\title{
Thymosin $\beta 4$ is essential for thrombus formation by controlling the G-actin/F-actin equilibrium in platelets
}

Inga Scheller, ${ }^{\star *}$ Sarah Beck, ${ }^{1 *}$ Vanessa Göb, ${ }^{1}$ Carina Gross, ${ }^{1}$ Raluca A. I. Neagoe, ${ }^{1,2}$ Katja Aurbach, ${ }^{1}$ Markus Bender, David Stegner, Zoltan Nagy ${ }^{1}$ and Bernhard Nieswandt

${ }^{1}$ Institute of Experimental Biomedicine I, University Hospital, University of Würzburg, and Rudolf Virchow Center for Integrative and Translational Biolmaging, University of Würzburg, Würzburg, Germany and ${ }^{2}$ Institute of Cardiovascular Sciences, College of Medical and Dental Sciences, University of Birmingham, Edgbaston, Birmingham, UK

*IS and SB contributed equally as co-first authors.
Correspondence: B. Nieswandt bernhard.nieswandt@virchow.uniwuerzburg.de

Received: $\quad$ February 11, 2021

Accepted: $\quad$ July 26, 2021.

Prepublished: August 5, 2021.

https://doi.org/10.3324/haematol.2021.278537

(อ2022 Ferrata Storti Foundation Published under a CC BY-NC license @ (1) $\Theta$

\begin{abstract}
Coordinated rearrangements of the actin cytoskeleton are pivotal for platelet biogenesis from megakaryocytes but also orchestrate key functions of peripheral platelets in hemostasis and thrombosis, such as granule release, the formation of filopodia and lamellipodia, or clot retraction. Along with profilin (Pfn) 1, thymosin $\beta 4$ (encoded by Tmsb4x) is one of the two main G-actin-sequestering proteins within cells of higher eukaryotes, and its intracellular concentration is particularly high in cells that rapidly respond to external signals by increased motility, such as platelets. Here, we analyzed constitutive Tmsb4x knockout (KO) mice to investigate the functional role of the protein in platelet production and function. Thymosin $\beta 4$ deficiency resulted in a macrothrombocytopenia with only mildly increased platelet volume and an unaltered platelet life span. Megakaryocyte numbers in the bone marrow and spleen were unaltered, however, Tmsb4x KO megakaryocytes showed defective proplatelet formation in vitro and in vivo. Thymosin $\beta 4$-deficient platelets displayed markedly decreased G-actin levels and concomitantly increased F-actin levels resulting in accelerated spreading on fibrinogen and clot retraction. Moreover, Tmsb4x KO platelets showed activation defects and an impaired immunoreceptor tyrosine-based activation motif (ITAM) signaling downstream of the activating collagen receptor glycoprotein VI. These defects translated into impaired aggregate formation under flow, protection from occlusive arterial thrombus formation in vivo and increased tail bleeding times. In summary, these findings point to a critical role of thymosin $\beta 4$ for actin dynamics during platelet biogenesis, platelet activation downstream of glycoprotein VI and thrombus stability.
\end{abstract}

\section{Introduction}

Platelets are small anucleate cells circulating in the blood stream that are essential for hemostasis and maintenance of vascular integrity but are also critically involved in thrombosis under pathological conditions. They are derived from giant precursor cells, the megakaryocytes (MK), residing in the bone marrow (BM). Mature polyploid MK extend long protrusions, so-called proplatelets, into the sinusoidal vessel lumen, which are shed off by shear forces and further fragment into platelets in the blood stream, ${ }^{1}$ a process that requires extensive microtubule and actin rearrangements. ${ }^{2,3}$ Whereas the actin cytoskeleton is thought to regulate proplatelet branching, microtubule sliding ensures proplatelet elongation. ${ }^{4}$ In circulating platelets, the actin cytoskeleton is essential to maintain cell morphology and to exert key functions upon activation, such as granule release, as well as the forma- tion of filopodia and lamellipodia. ${ }^{5}$ The critical role of the actin cytoskeleton for platelet production and function is demonstrated by platelet disorders in humans and mice resulting from defects in proteins regulating actin dynamics. ${ }^{6-8}$ However, the complex protein network orchestrating actin dynamics in $\mathrm{MK}$ and platelets remains incompletely understood.

$\beta$-thymosins are a family of proteins with a molecular weight of approximately $5 \mathrm{kDa}$ that are widely expressed. Of the 15 existing, highly homologous $\beta$-thymosins, thymosin $\beta 4$ is the most abundant isoform. ${ }^{9}$ Thymosin $\beta 4$ was first isolated from calf thymus and therefore thought to exert hormone-like activities. ${ }^{10,11}$ Indeed, various paracrine effects of the protein have been reported, including cardiac protection, angiogenesis, wound healing and immunomodulatory effects ${ }^{9,12-14}$ although the exact underlying mechanisms have not been fully elucidated.

The intracellular concentration of thymosin $\beta 4$ is particu- 
larly high in cells that rapidly respond to external signals by increased motility such as neutrophils and macrophages or by profound shape changes like platelets $(\sim 140,000$ copies per platelet in mice and 320,000 copies per platelet in humans). ${ }^{15-17}$ For the dynamic spatiotemporal regulation of globular $(G)$-actin polymerization into filamentous (F)-actin networks, cells rely on a large reservoir of actin monomers sequestered by the actin monomerbinding proteins thymosin $\beta 4$ and profilin (Pfn) $1 .^{18}$ The main intracellular function of thymosin $\beta 4$ is to bind G-actin, thereby inhibiting actin polymerization. ${ }^{19}$ Thymosin $\beta 4$ exclusively binds to actin monomers and not to the filament ends or alongside the filament, and its affinity is 50-fold higher for ATP- than ADP-bound actin monomers maintaining a polymerization-ready pool in reserve. ${ }^{20,21}$ In resting cells, thymosin $\beta 4$ complexes about half of the total G-actin. ${ }^{22}$ Whereas thymosin $\beta 4$ prevents actin polymerization, Pfn1 promotes filament assembly. ${ }^{19}$ Both proteins bind actin monomers transiently with a stoichiometry of 1:1.23 While Pfn1 catalytically accelerates nucleotide exchange, thymosin $\beta 4$ strongly inhibits the exchange of the nucleotide bound to actin monomers by blocking its dissociation. ${ }^{23}$

Conditional MK- and platelet-specific Pfn1 knockout (KO) mice reproduced key features of Wiskott-Aldrich syndrome (WAS) patients including microthrombocytopenia, due to impaired proplatelet formation (PPF), cytoskeletal alterations and accelerated platelet clearance. ${ }^{24}$ Pfn1 KO MK produced less and smaller-sized platelets into the circulation, which had a thicker marginal band and a partially disrupted actin cytoskeleton leading to accelerated integrin inactivation and hence impaired platelet function in vitro and in vivo. ${ }^{25}$

As dynamic actin reorganization is crucial for both platelet biogenesis and function, ${ }^{24-26}$ we investigated the role of thymosin $\beta 4$ in these processes by studying Tmsb4x KO mice. Deficiency of thymosin $\beta 4$ resulted in macrothrombocytopenia and defective proplatelet formation due to a dysregulated G- to F-actin ratio. Moreover, Tmsb4x KO platelets displayed impaired mmunoreceptor tyrosine-based activation motif (ITAM) signaling downstream of glycoprotein $\mathrm{VI}$ (GPVI), leading to defective aggregate formation under flow, protection from in vivo thrombus formation, and increased tail bleeding times.

\section{Methods}

\section{Animals}

All animal studies were approved by the district government of Lower Franconia (Bezirksregierung Unterfranken). Tmsb4 $\mathrm{X}^{-1}$ mice were created by MRC mouse network by injection of embryonic stem cell clone Tmsb4 $4 x^{\text {tm2a(EUсомM)Wtsi }}$ into C57Bl/6/129/SvJ blastocysts and afterwards trans- ferred to our animal facility where the KO mice were kept on the mixed background. Eight to 16-week-old KO mice and matching wild-type (WT) animals were used for experiments if not stated otherwise.

Detailed protocols for platelet preparation, determination of platelet lifespan, count and size, aggregometry, flow adhesion assays, actin polymerization, tail bleeding time, intravital microscopy models, two-photon microscopy, spreading assays and clot retraction, as well as MK differentiation and culture, histology, staining procedures and immunoblotting can be found in the Online Supplementary Appendix.

\section{Data analysis}

The presented results are mean \pm standard deviation (SD) from at least three independent experiments per group, if not stated otherwise. Data distribution was analyzed using the Shapiro-Wilk-test and differences between control and KO mice were statistically analyzed using unpaired, two-tailed Student's $t$-test, one-way ANOVA or Fisher's exact test as indicated in the legends. $P$-values $<0.05$ were considered as statistically significant, ${ }^{*} P<0.05$; $* * P<0.01$; $\star * * P<0.001$. Results with a $P$-value $>0.05$ were considered as not significant (ns).

\section{Results}

\section{Impaired proplatelet formation and} macrothrombocytopenia in thymosin $\beta 4$-deficient mice Constitutive Tmsb4x KO mice were born in the expected Mendelian ratios, viable and fertile. The complete absence of thymosin $\beta 4$ in platelets was confirmed by an automated quantitative capillary-based immunoassay platform, Jess (Figure 1A). Tmsb4x KO mice showed no change in basal blood parameters except for platelet count and size (Online Supplementary Table S1). Platelet counts were significantly reduced in Tmsb4x KO mice compared to WT controls (Figure 1B). Mutant platelets exhibited a small, but significant increase in mean platelet volume (Figure 1C). Transmission electron microscopy (TEM) images revealed that platelet size was quite variable in mutant mice, with platelets comparable to WT size as well as big roundish platelets and platelets with normal shape but increased size (Figure 1D). $\alpha$ - and dense granule numbers were comparable between WT and Tmsb4x KO platelets (Online Supplementary Figure S1A and B). Notably, platelet lifespan in Tmsb4x KO mice was unaltered as compared to the WT controls (Online Supplementary Figure S2A). Moreover, analysis of hematoxylin and eosin-stained (H\&E) femora and spleen sections (Figure 1E and F; Online Supplementary Figure $S 2 B$ ) as well as cryosections of femora (Online Supplementary Figure $S 2 C$ and $D$ ) revealed comparable MK numbers in WT and Tmsb4x KO mice. MK ploidy 
A

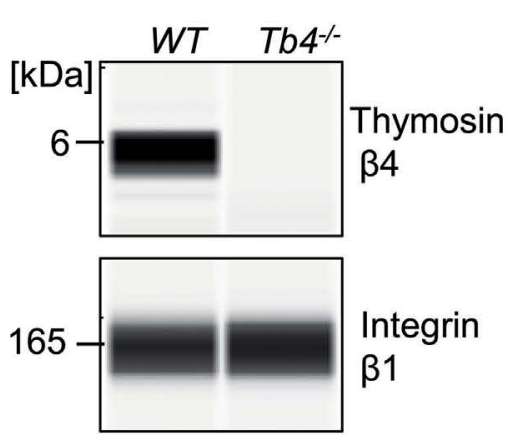

D

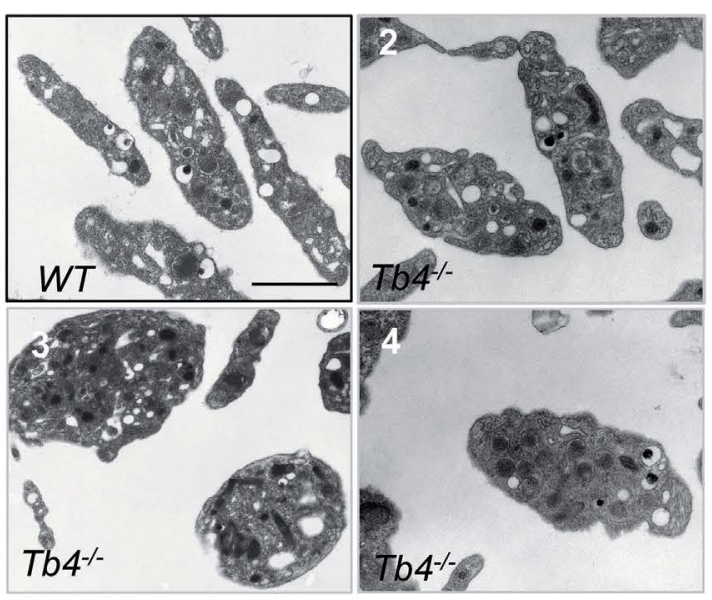

G
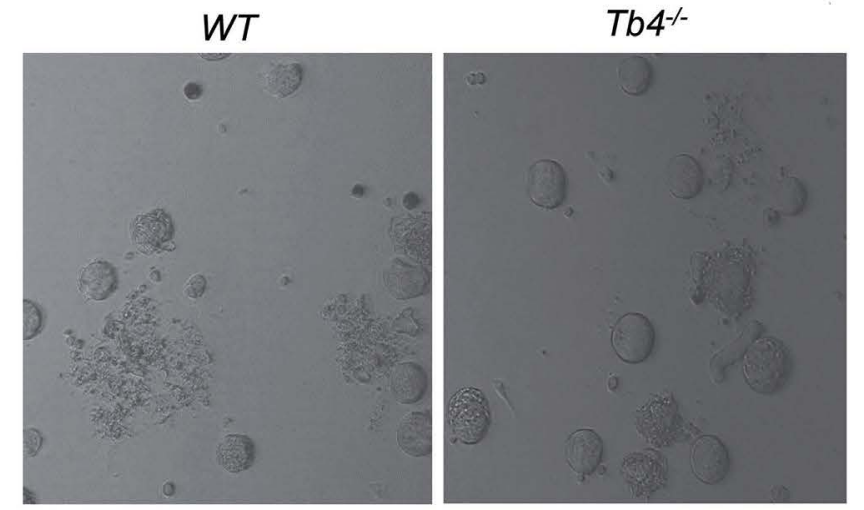

E

5

客

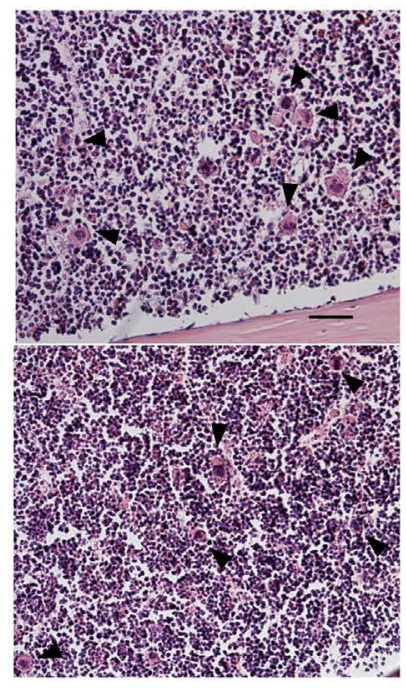

C

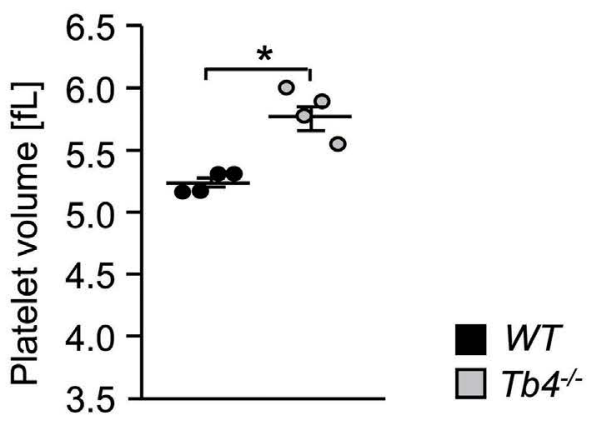

H

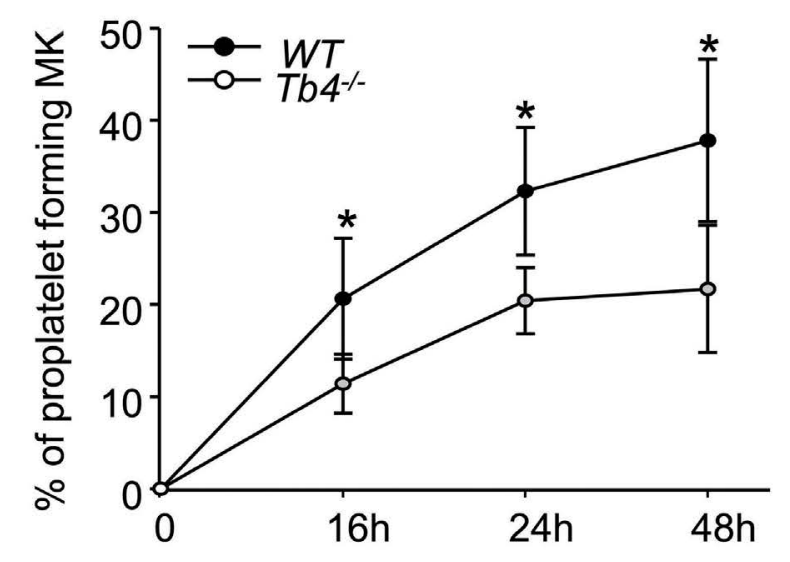

I
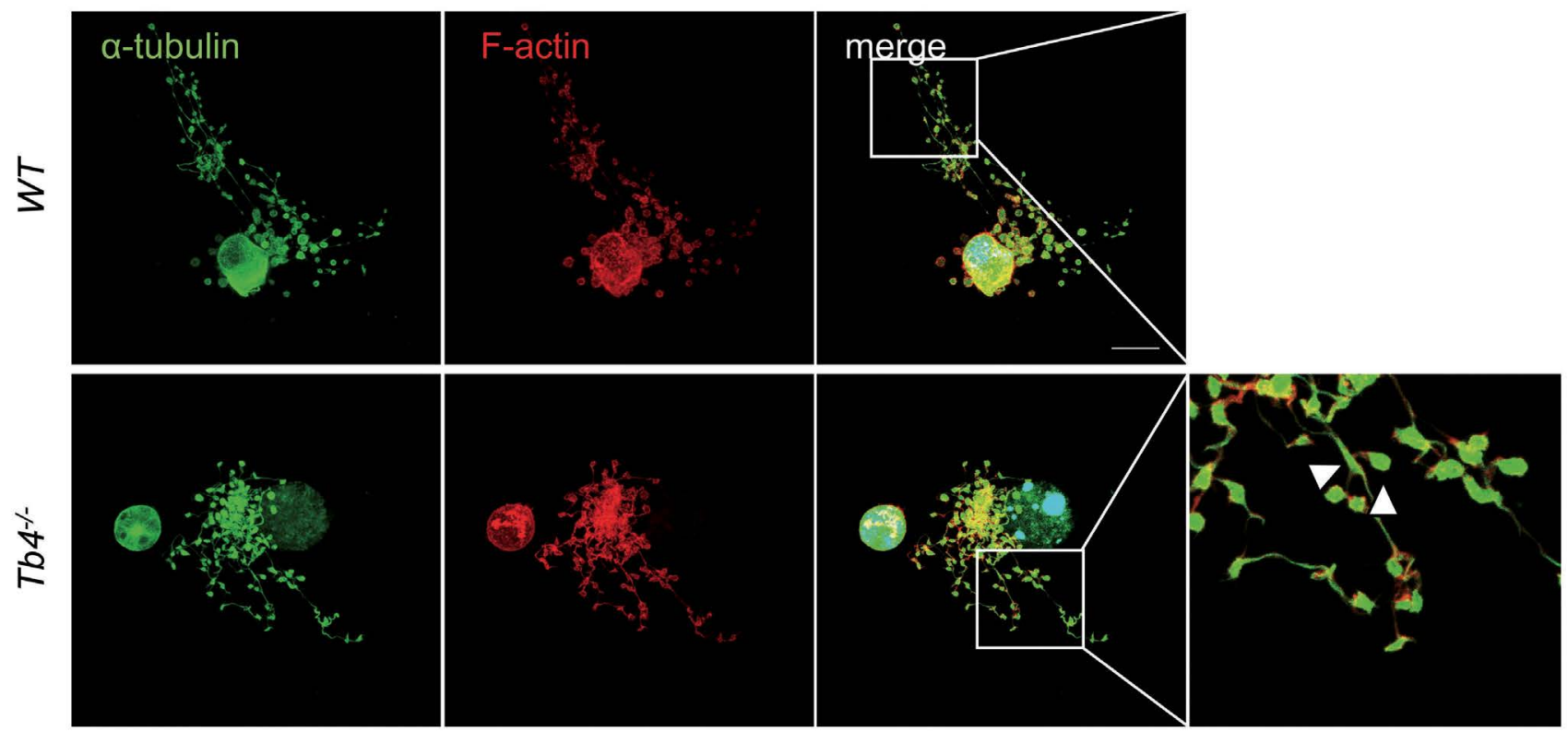

Figure 1. Thrombocytopenia and impaired proplatelet formation in thymosin $\beta 4$ knockout mice. (A) Protein levels of thymosin $\beta 4$ and integrin $\beta 1$ in wild-type (WT) and Tmsb4x knockout (KO) platelets were analyzed by an automated quantitative capillarybased immunoassay platform; Jess (Proteinsimple). Platelet count (B) and volume (C) were determined using an automated blood cell analyzer (ScilVet). Mean \pm standard deviation (SD) ( $n=4,3$ independent experiments). Unpaired, two-tailed Student's $t$-test. $* * \star P<0.001$. (D) Representative transmission electron microscopic images of 1 WT mouse and 3 Tmsb4x KO mice: (2) platelets comparable to WT size, (3) big roundish platelets, (4) platelets with increased size. Scale bars: 2 um. (E and F) Hematoxylin-eosin stainings of femur paraffin sections of WT and Tmsb4x KO mice (E) and quantification of megakaryocyte (MK) numbers (F). Arrow heads indicate the MK. Scale bars: $100 \mu \mathrm{m}$. Values are mean \pm SD $(n=3)$. $(G$ and $H)$ Proplatelet formation of bone marrow MK after lineage depletion and culturing in rHirudin- and TPO-conditioned medium. On day 4, proplatelet-forming MK were counted. Average of 5 analyzed visual fields per MK culture of 3 animals/genotype is shown. Values are mean \pm SD. Unpaired, two-tailed Student's $t$-test. ${ }^{\star} P<0.05$. (I) Proplatelets were visualized using an $\alpha$-tubulin antibody and phalloidin and analyzed by confocal microscopy (40x objective, Leica TCS SP8) using a 40x objective. Scale bar: $20 \mu \mathrm{m}$. 
levels in mutant mice were not significantly altered compared to WT animals as assessed by flow cytometric analysis of freshly isolated BM MK (Online Supplementary Figure $S 2 E)$. TEM of BM MK revealed a slightly altered demarcation membrane system (DMS) morphology with more and smaller invaginations (Online Supplementary Figure S3).

In order to elucidate whether the macrothrombocytopenia in Tmsb4x KO mice was caused by impaired platelet biogenesis, we analyzed the ability of $\mathrm{BM}$-derived $\mathrm{MK}^{27}$ to form proplatelets in vitro. We found that PPF was significantly reduced in the absence of thymosin $\beta 4\left({ }^{\star} P<0.05\right)$ (Figure $1 G$ and $H$ ). Microscopic analysis of proplatelet-forming MK revealed the presence of thickened and shortened proplatelet shafts and tips, suggesting that defective cytoskeletal organization may account for the reduced PPF (Figure 11). In order to visualize PPF in vivo, we imaged MK in the BM of WT and Tmsb4x KO mice by two-photon intravital microscopy of the skull. As shown in the Online Supplementary Video S1, proplatelet-forming MK in WT mice formed protrusions reaching into the vessel sinusoids that were rapidly shed off by the blood flow (Online Supplementary Video S1). In marked contrast, large, abnormally thick protrusions were observed in Tmsb4x KO mice (Online Supplementary Video S2). Interestingly, these aberrant proplatelets dissociated less frequently from the MK and appeared to be more firmly attached to its mother cell (Online Supplementary Video S2). Notably, we did not observe ectopic release of proplateletlike particles into the BM, as previously reported for other proteins involved in actin dynamics such as $P f n 1,{ }^{24} A D A P^{28}$ or WASP ${ }^{29} \mathrm{KO}$ mice. This suggests that thymosin $\beta 4$ deficiency induces pronounced cytoskeletal defects resulting in impaired platelet biogenesis in vivo but not ectopic platelet release into the BM compartment. Actomyosin contractility plays a crucial role for the fragmentation of protrusions from MK into the blood stream..$^{30}$ Thus, we assessed myosin levels in MK and platelets. Although non-muscle myosin IIA (NMIla) levels were comparable in MK (Online Supplementary Figure S4A), Tmsb4x KO platelets displayed significantly decreased NMIla (Online Supplementary Figure $S 4 B$ and $C$ ) and myosin light chain 2 (MLC2; Online Supplementary Figure $S 4 D$ and $E$ ) levels compared to WT. These findings indicate that in the absence of thymosin $\beta 4$, myosin enrichment during the late stages of $\mathrm{MK} /$ proplatelet maturation is affected. This, together with the disturbed actin dynamics, which play a crucial role for proplatelet branching, ${ }^{1}$ might explain the existence of abnormal, elongated proplatelets without swellings in Tmsb4x KO mice.

\section{Defective actin dynamics in thymosin $\beta 4$-deficient platelets}

As thymosin $\beta 4$ is one of the major G-actin sequestering proteins, we analyzed F-actin content and found that resting Tmsb4x KO platelets exhibited a significant increase in $F$-actin levels, as assessed by flow cytometry using fluor- escently labeled phalloidin ( $\left.{ }^{*} P<0.05\right)$ (Figure $\left.2 A\right)$. Strikingly, Tmsb4x KO platelets were unable to efficiently assemble further F-actin upon activation with different agonists (Figure $2 \mathrm{~B}$ ). Furthermore, by separating the actin cytoskeleton into monomeric and polymeric fractions using ultracentrifugation, ${ }^{24}$ we observed reduced actin protein levels in the supernatant fraction of Tmsb4x KO platelets indicating lower G-actin levels compared to WT platelets $(* * P<0.01)$ (Figure $2 \mathrm{C}$ and $\mathrm{D}$ ). In line with these observations, G-actin content was reduced by $50 \%$ in $T m s b 4 x$ KO platelets spread on fibrinogen as determined by staining with DNase I to label G-actin ${ }^{31}(* * P<0.01)$ (Figure 2E and F).

As Pfn1 might functionally compensate for the loss of thymosin $\beta 4$, we determined Pfn1 protein levels by western blotting, and found them unaltered compared to the WT. Moreover, assessment of the activation-dependent phosphorylation of Pfn1 on tyrosine 129, which is known to increase the affinity of Pfn1 towards actin monomers and its actin polymerization activity, revealed an unaltered activity of Pfn1 (Online Supplementary Figure S5).

\section{Accelerated spreading and clot retraction of thymosin $\beta 4$-deficient platelets}

Platelet spreading on fibrinogen is highly dependent on functional cytoskeletal dynamics. Therefore, we next assessed the ability of Tmsb4x KO platelets to form filo- and lamellipodia on a fibrinogen matrix. As shown in Figure $3 \mathrm{~A}$ to $C$, we found overall accelerated spreading of KO platelets, which was most evident at the 15-minute time point. We speculate that the formation of protrusions in the $\mathrm{KO}$ platelets is facilitated due to the increased F-actin content. We further analyzed the actin filaments of spread platelets on fibrinogen using phalloidin-647 and an anti-tubulin-488 antibody staining (Online Supplementary Figure S6) or platinum replica electron microscopy (PREM) (Figure $3 C$ and D). Of note, we compared earlier time points of spread Tmsb4x KO with later time points of WT platelets to compensate for the faster spreading.

When comparing WT platelets spread for 15 minutes (min) with $T m s b 4 x \mathrm{KO}$ platelet spread for $5 \mathrm{~min}$ in the PREM assay (or 30 min WT to 15 min Tmsb4x KO for the staining), we found unaltered F-actin structures. Strikingly, clot retraction of Tmsb4x KO platelets was also enhanced (Figure 4A and B), indicating faster contraction of the actin cytoskeleton, generating forces transmitted to the external fibrin clot, resulting in retraction and fibrin clot shrinkage. In order to further analyze the dynamics of clot retraction, we performed immunofluorescence staining of the fibrin meshwork of the clots. ${ }^{32}$ Clots were analyzed by confocal microscopy and strikingly, as shown in Figure 4C, Tmsb4x KO platelets displayed less and shorter fibrin fibers and they also look less branched. These findings indicate that Tmsb4x KO platelets are able to bend and shorten fibrin fibers faster than WT pla- 
telets, which might explain, or at least contribute to, the observed faster clot retraction.

\section{Defective activation and aggregation of thymosin $\beta 4$-deficient platelets}

Next, we sought to investigate the effect of thymosin $\beta 4$ deficiency on platelet activation. Washed platelets were stimulated with various agonists and the activation of $\alpha$ llb $\beta 3$ integrin (JON/A-PE) as well as degranulation-dependent surface exposure of P-selectin was determined. Although expression levels of prominent surface glycoproteins were comparable between mutant and WT platelets (Online Supplementary Table S2), slightly reduced $\alpha$ llb $\beta 3$ activation was consistently detected in Tmsb4x KO platelets in response to stimulation with ADP, thrombin and the snake venom toxin rhodocytin which activates the hemITAM coupled C-
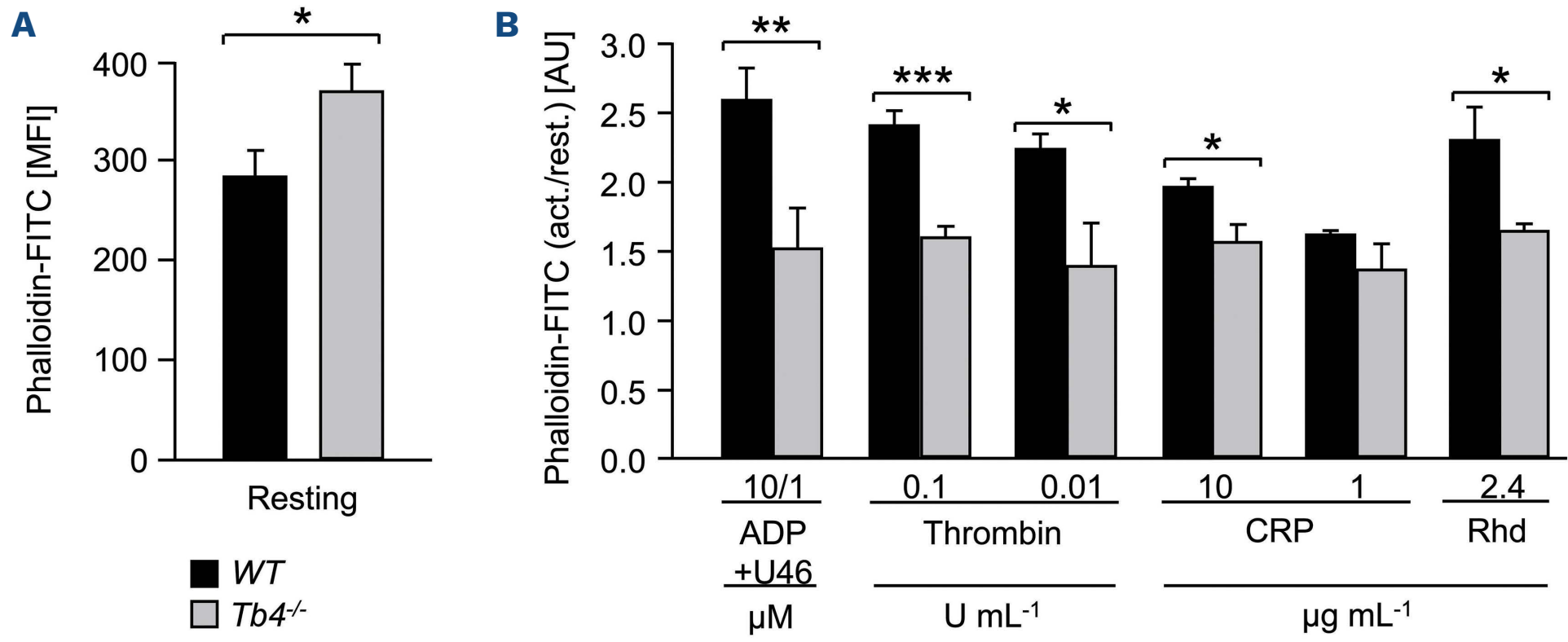

C

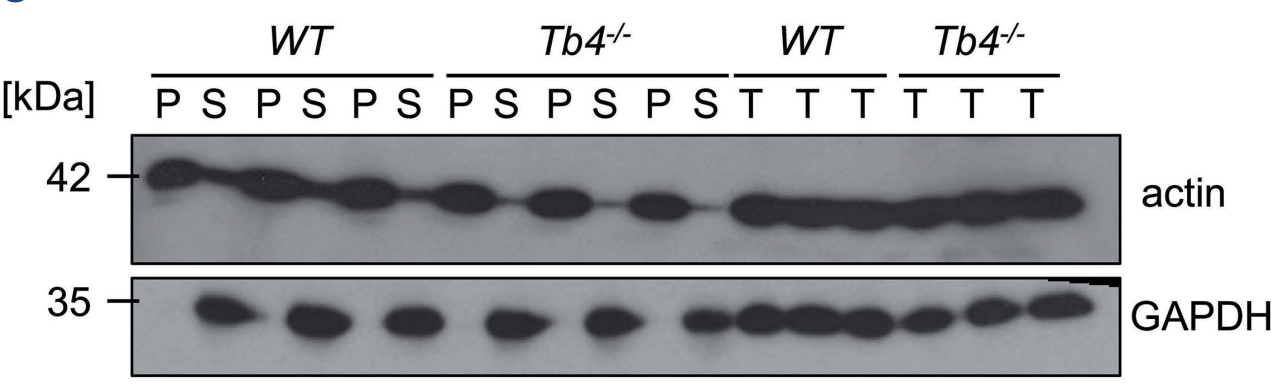

*P: Pellet; S: Supernatant; T: Total
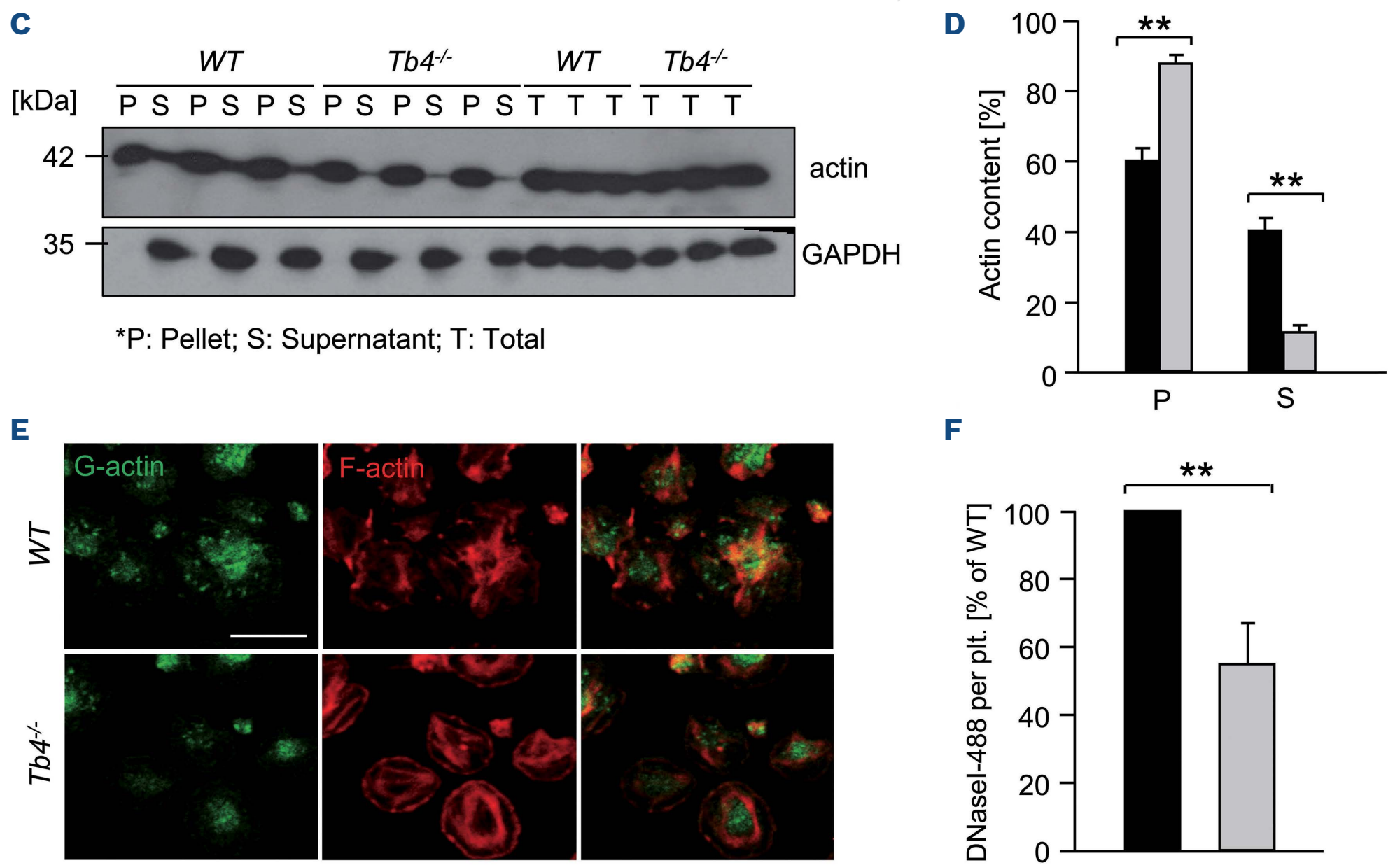

$\mathbf{F}$

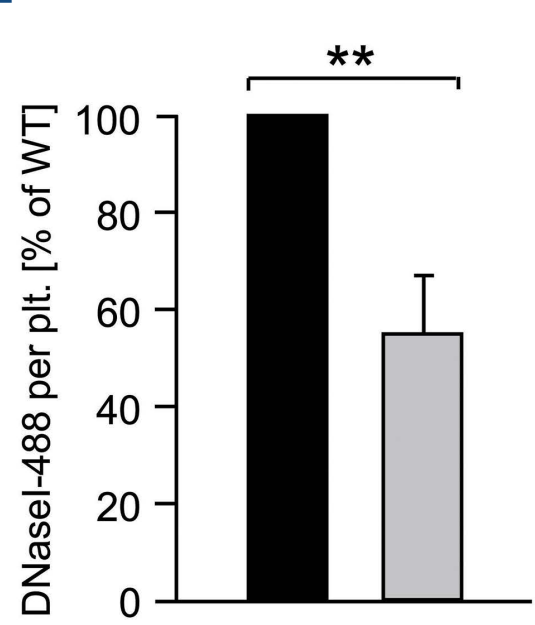

Figure 2. Impaired actin equilibrium and assembly in thymosin $\beta 4$ knockout platelets. (A and B) Relative F-actin content of resting and activated platelets was determined by flow cytometry. Values are mean \pm standard deviation (SD) of 4 mice per group. The values are displayed as the ratio of MFI: mean fluorescence intensity from activated and resting platelets. Unpaired, two-tailed Student's t-test. $* P<0.05, * * P<0.005, * \star * P<0.001$. (C and D) The actin cytoskeleton was isolated by ultracentrifugation, immunoblotted with an anti- $\beta$-actin antibody and analyzed for the content of monomeric vs. filamentous actin using densitometry. GAPDH served as loading control. Values are mean \pm SD $(n=3)$. P: pellet, S: supernatant, T: total protein. Unpaired, two-tailed Student's $t$-test. ${ }^{*} P<0.005$. ( $E$ and $F$ ) Visualization of the cytoskeleton of spread platelets (15 minutes) on fibrinogen, which were stained with DNase I-AlexaF488 (green) to label G-actin and Phalloidin-atto647N (red) for visualization of F-actin and analyzed by confocal microscopy. Scale bar: $20 \mu \mathrm{m}$. Values are mean \pm SD $(n=3)$. Unpaired, two-tailed Student's $t$-test. $* * P<0.005$. 
lectin like receptor 2 (CLEC-2), whereas the response to the GPVI-specific agonist, collagen-related peptide (CRP), was strongly reduced (Figure 5A). In order to exclude pre-activation of Tmsb4x KO platelets, we performed fluorescenceactivated cell sorting (FACS) analysis of JON/A-PE binding upon stimulation with epinephrine (Online Supplementary Figure S7) showing no pre-activation.

$\mathrm{P}$-selectin expression was also reduced in response to CRP, but not in response to other agonists, in Tmsb4x KO platelets, even at high agonist concentrations (Figure

A

Fibrinogen

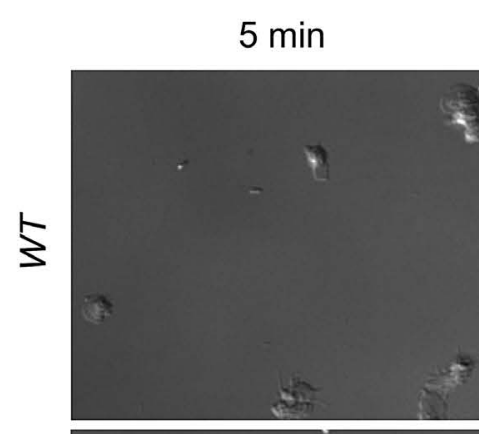

$15 \min : K O$

$30 \mathrm{~min}: \mathrm{WT}+\mathrm{KO}$
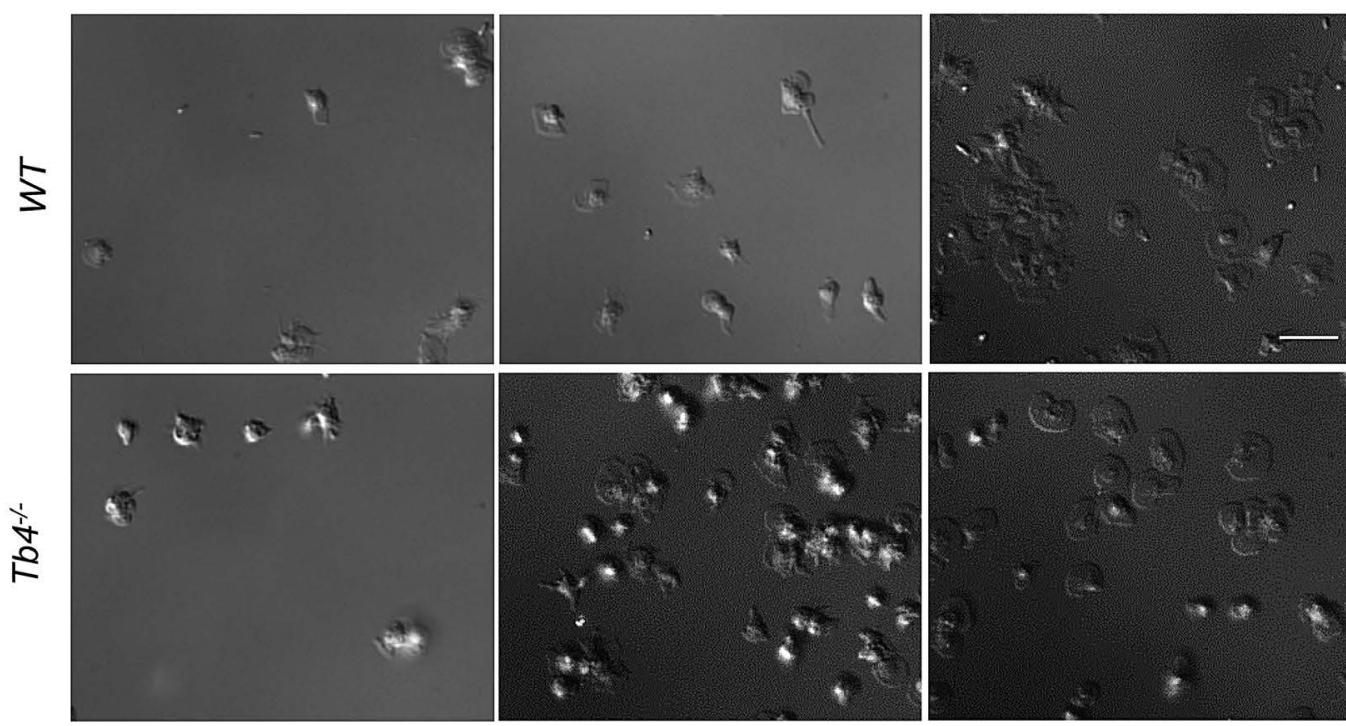

$\mathbf{B}$
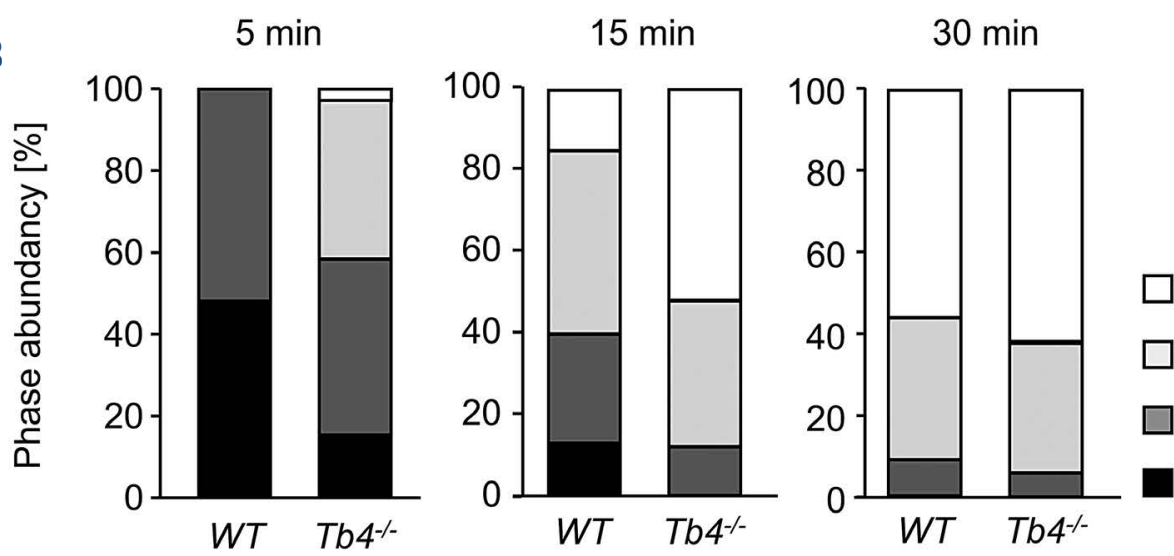

fully spread filo- and lamellipodia filopodia

resting

C

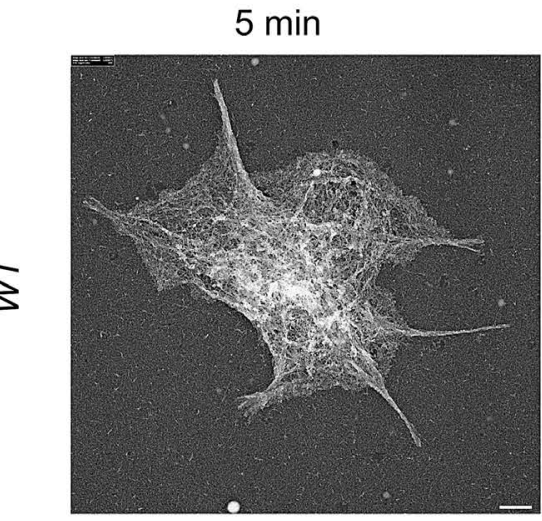

D
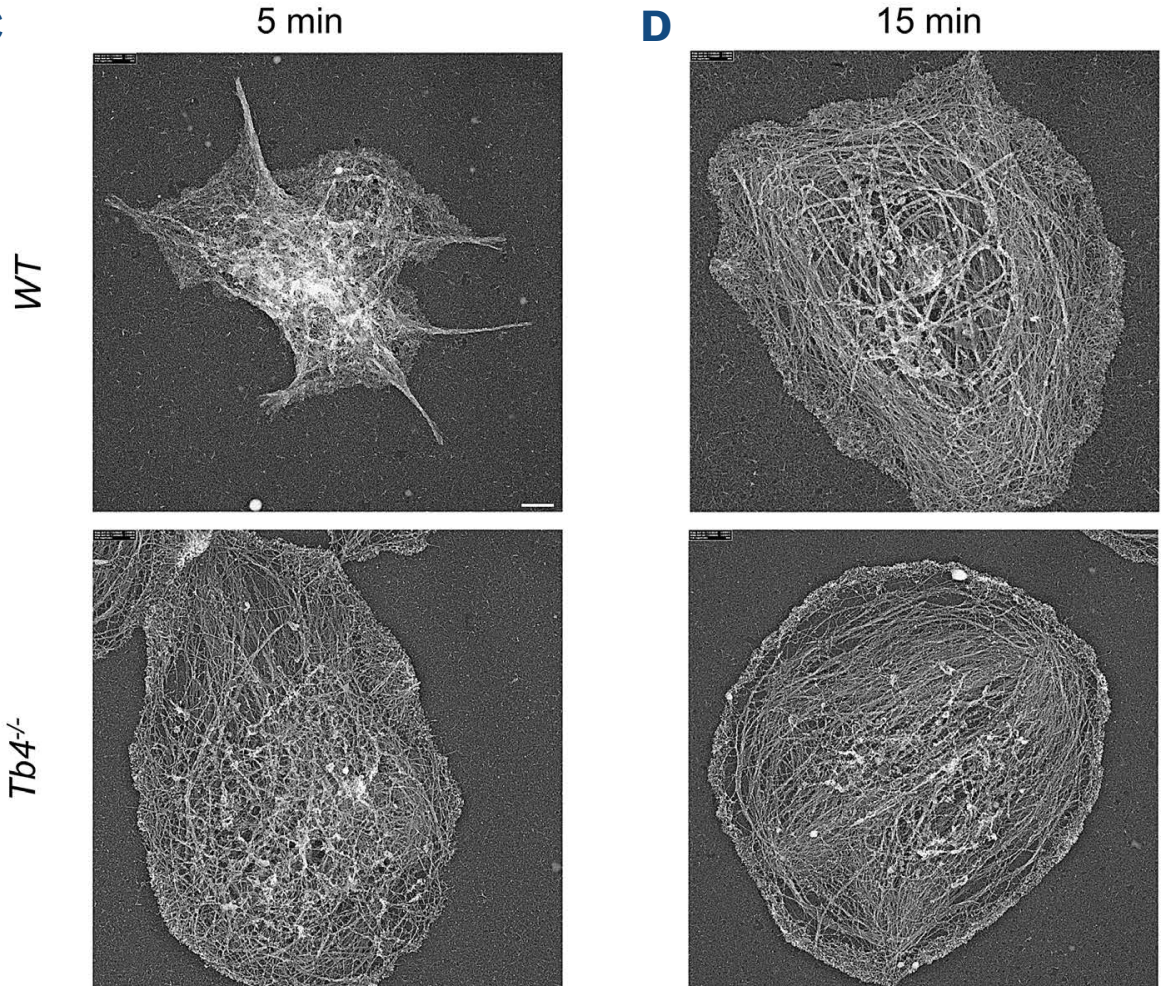

Figure 3. Accelerated spreading of thymosin $\beta 4$ knockout platelets. ( $A$ and $B$ ) Washed platelets were stimulated with $0.01 \mathrm{U} \mathrm{L}^{-}$ ${ }^{1}$ thrombin and allowed to spread $\left(5,15,30\right.$ minutes [min]) on fibrinogen (100 $\mu \mathrm{g} \mathrm{mL} \mathrm{mL}^{-1}$. DIC pictures were taken (A) and phase abundance was determined (B). Images are representatives of at least 6 animals per group. Scale bar: 3 um. (C and D) Representative images of the platelet cytoskeleton ultrastructure of wild-type (WT) and Tmsb4x knockout (KO) mice on fibrinogen after $5 \mathrm{~min}(\mathrm{C})$ and $15 \mathrm{~min}(\mathrm{D})$. Scale bar: $500 \mathrm{~nm}$. 
5B). This selective secretion defect was also confirmed when ATP release was determined by luminoaggregometry. In response to thrombin and U46619 (a stable thromboxane analogue), ATP release was slightly increased in Tmsb4x KO platelets compared to WT. In contrast, ATP release in response to CRP was significantly reduced in the mutant platelets (e.g., $62 \pm 2.5 \mu \mathrm{M}$ in WT vs. $25 \pm 4 \mu \mathrm{M}$ in Tmsb4x $\mathrm{KO}$ for $0.1 \mathrm{mg} \mathrm{mL}^{-1} \mathrm{CRP}$, $\star \star \star P<0.001$ ) (Figure $5 \mathrm{C}$ ).

Tmsb $4 x$ KO platelets also exhibited a marked aggregation defect upon thrombin and CRP as well as convulxin (CVX) stimulation, whereas aggregation in response to ADP and U46619 stimulation was unaltered (Figure 5D). Of note, ag- gregation responses of Tmsb4x KO platelets following stimulation of CLEC-2 were comparable to WT platelets (Online Supplementary Figure S8). Analysis of GPVI signaling revealed a reduced phosphorylation of the FCRy chain in the mutant platelets (Figure 5E). Moreover, Jess analysis showed decreased phosphorylation of the tyrosine kinase Syk in mutant platelets, indicating a very early signaling defect downstream of GPVI that explains the in vitro platelet defects (Figure 5F and G). Of note, we observed an increase in total Syk protein levels in Tmsb4x KO platelets using the Jess assay where platelet lysates are centrifuged before immunoblotting, however, when analyzing whole platelet lysates, we could not detect differences in total Syk levels (data not shown).
A

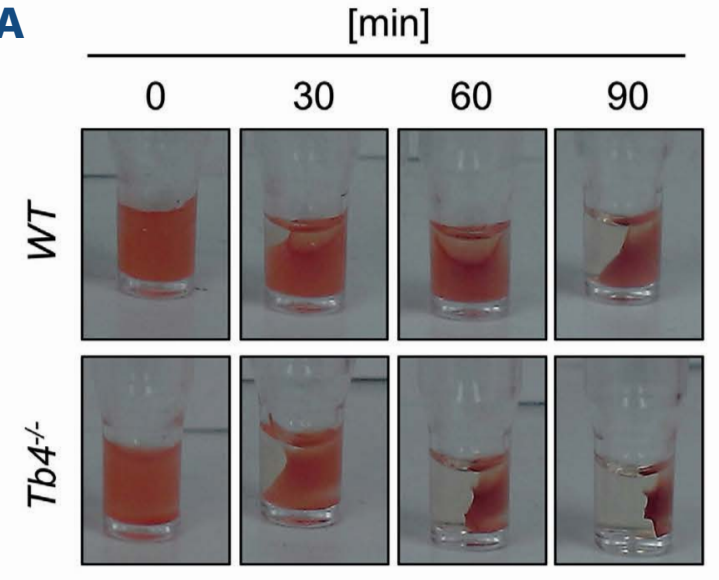

C

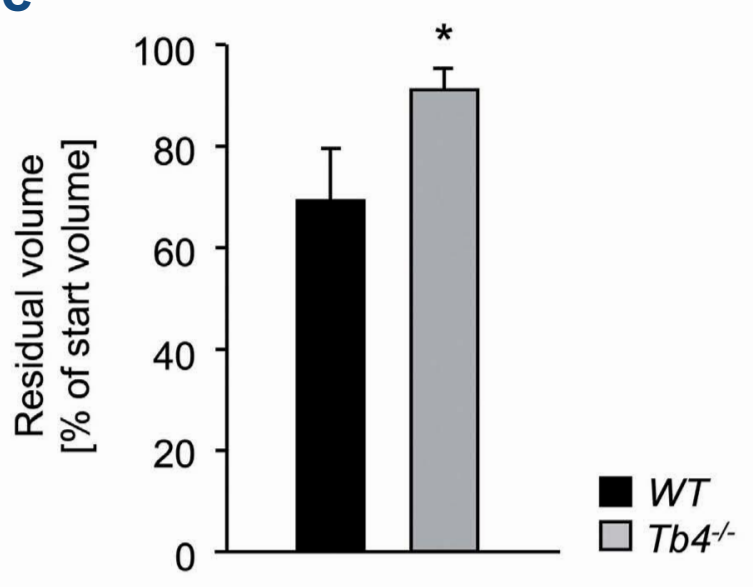

B
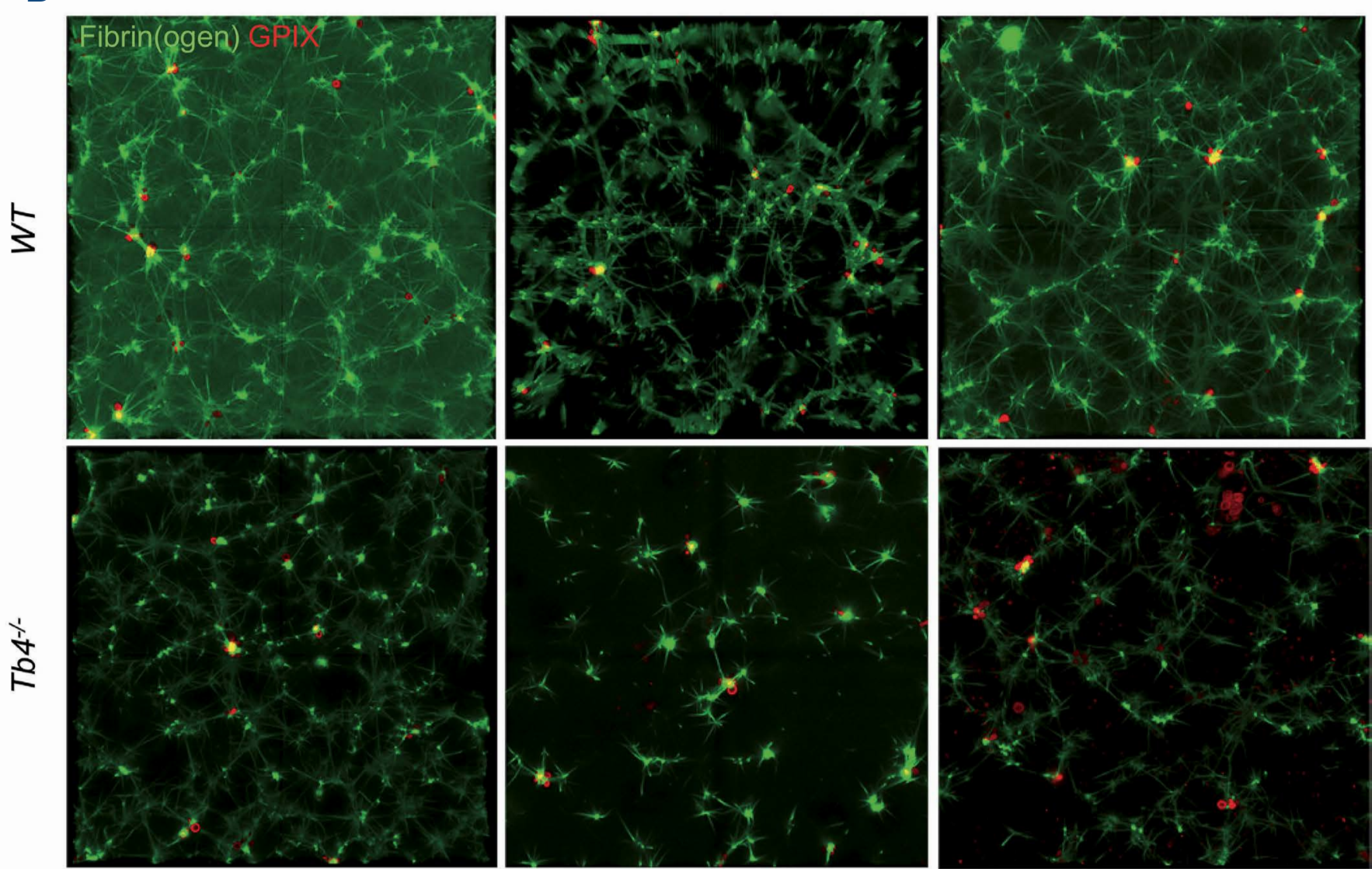

Figure 4. Accelerated clot retraction of thymosin $\beta 4$ knockout platelets. (A) Clot retraction of wild-type (WT) and Tmsb4x knockout (KO) platelet-rich plasma (PRP) was determined in response to $4 \mathrm{U} \mathrm{mL}^{-1}$ thrombin and monitored over time. (B) Residual volume at the end of the experiment. Values are mean \pm standard deviation (SD) ( $n=6$ per group). Unpaired, two-tailed Student's $t$-test. ${ }^{*}<0.05$. (C) Analysis of the fibrin meshwork of WT and Tmsb4x KO clots. Washed platelets were labeled with an anti-GPIX Alexa 647 derivative and added to a mix of unlabeled fibrinogen $\left(2 \mathrm{mg} \mathrm{mL}^{-1}\right)$ and Alexa Fluor A488-labeled fibrinogen $(50 \mu \mathrm{g} \mathrm{mL}$ ${ }^{1}$ f.c.). Platelets were stimulated with $0.1 \mathrm{U} \mathrm{mL}^{-1}$ thrombin and clotting was initiated by addition of $5 \mathrm{mM} \mathrm{CaCl}$. The mixture was immediately transferred to an uncoated 8-well chamber slide (Ibidi), and allowed to clot. Images were obtained using a Leica SP8 inverted microscope with a 63x oil immersion lens. Optical z-stacks were deconvolved and are shown as maximum projection. Images are representatives of at least 2 z-stacks per mouse and 4 animals per group. 
A

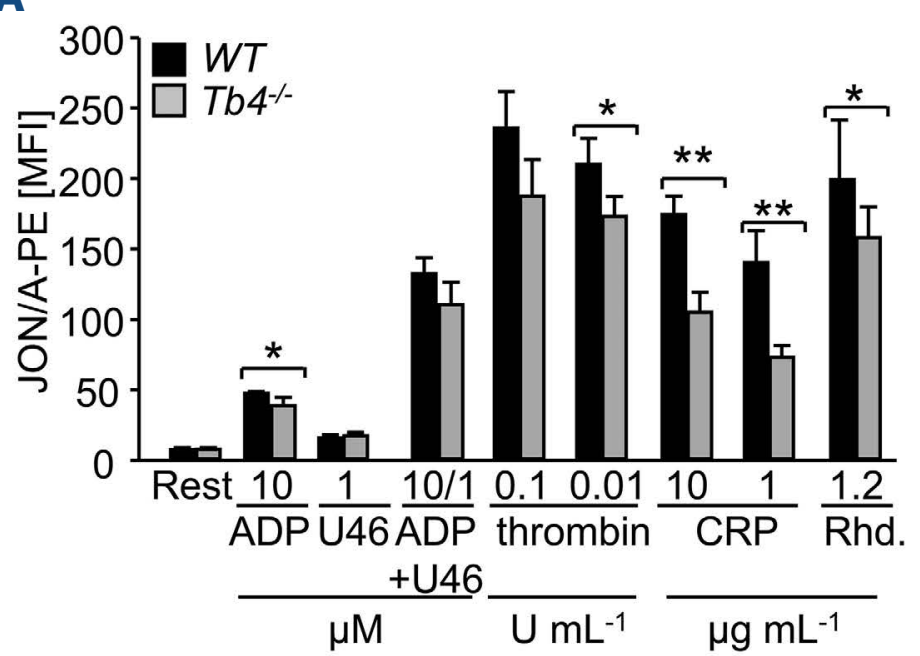

C

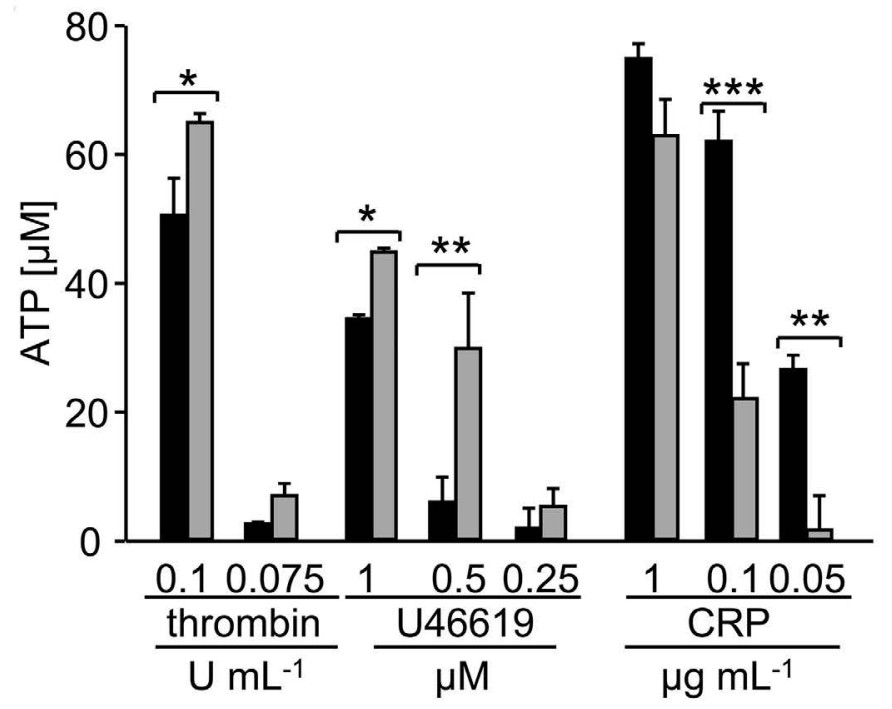

E

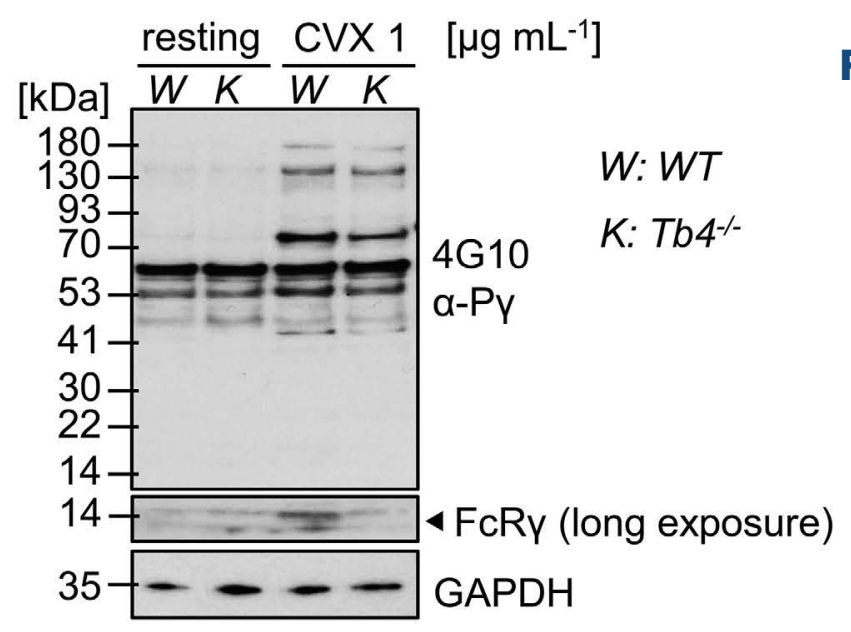

G

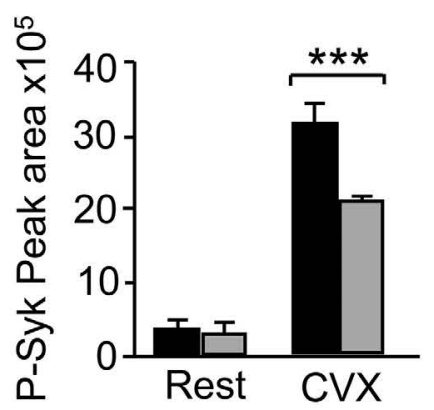

B

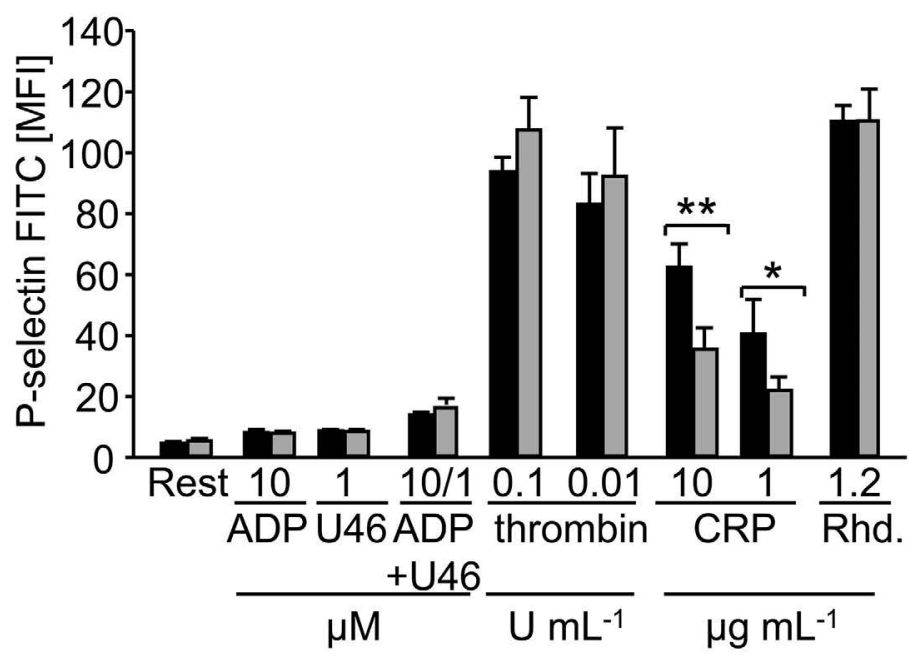

D

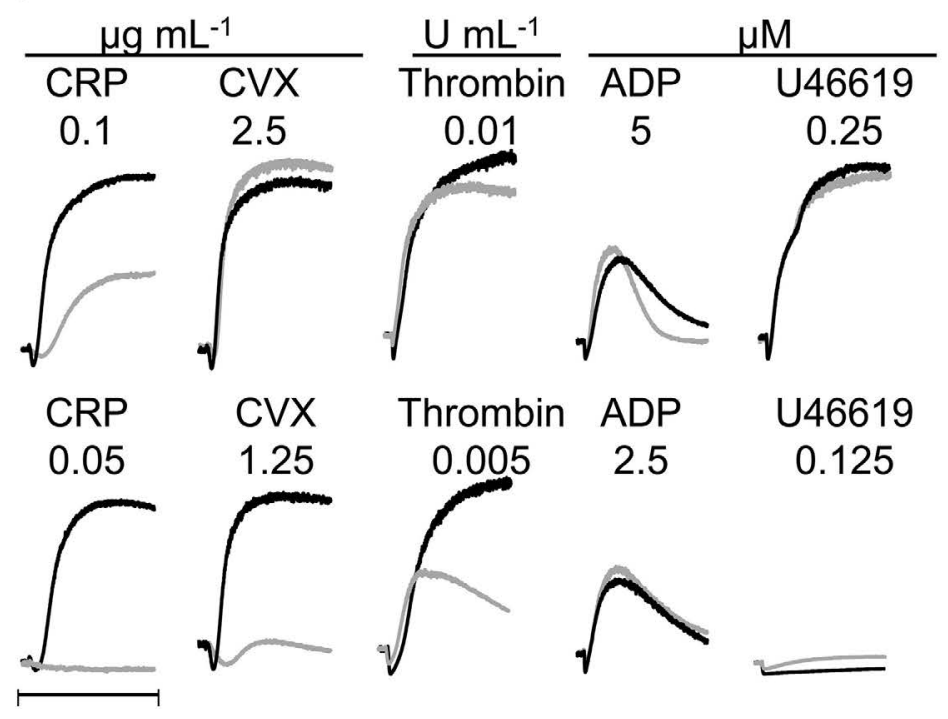

$\mathbf{F}$

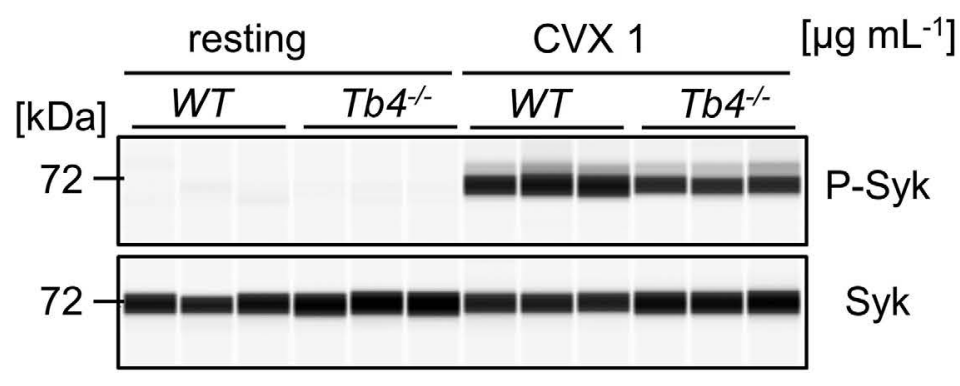

Figure 5. Altered $\alpha$ llb $\beta 3$ integrin activation, degranulation and aggregation of thymosin $\beta 4$ knockout platelets. (A and $B$ ) Activation of platelet $\alpha$ llb $\beta 3$ integrin (JON/A-PE) (A) and degranulation ( $\alpha$-P-selectin-FITC) (B) in wild-type (WT) and Tmsb4x knockout (KO) platelets upon stimulation with the indicated agonists was determined by flow cytometry ( $\mathrm{n}=12$ ). U46: U46619; CRP: collagen-related peptide; Rhd: rhodocytin. Unpaired, two-tailed Student's $t$-test. $* P<0.05, * \star P<0.005$. (C) Dense granule secretion was assessed by luminometric measurement of released ATP of activated WT and Tmsb4x KO platelets. Results are given as mean ATP concentration $[\mu \mathrm{M}] \pm$ standard deviation (SD) ( $\mathrm{n}=12$ per group). Unpaired, two-tailed Student's $t$-test. ${ }^{*} P<0.05$, $\star * P<0.005, * * * P<0.001$. (D) Aggregation responses of washed platelets or platelet-rich plasma (PRP) in turbidometric aggregometry $(n=6)$. (E) Western blot analysis of phosphotyrosine levels in resting and CVX-stimulated WT and Tmsb4x KO platelets using the 4 G10 antibody. GAPDH served as loading control. CVX: convulxin; K: Tb4 ${ }^{-/}$; W: WT. (F and G) Phosphorylation and total protein levels of Syk in resting and CVX-stimulated WT and Tmsb4x KO platelets were analyzed (E) and quantified (F) by an automated quantitative capillary-based immunoassay platform. Values are mean \pm SD $(n=3)$. Unpaired, two-tailed Student's $t$-test. ${ }^{*} P<0.05, * * P<0.005, * * * P<0.001$. MFI: mean fluorescence intensity. 


\section{Thymosin $\beta 4$ is required for thrombus formation under} flow conditions

In order to test the functional consequences of thymosin $\beta 4$ deficiency under more physiological conditions, we analyzed platelet adhesion and thrombus formation on a collagen-coated surface under flow in a whole-blood perfusion system. Under high $\left(1,700 \mathrm{~s}^{-1}\right)$, intermediate $\left(1,000 \mathrm{~s}^{-1}\right)$, and low $\left(150 \mathrm{~s}^{-1}\right)$ shear conditions, WT platelets rapidly adhered to the collagen surface and recruited additional platelets from the blood stream resulting in the formation of stable three-dimensional aggregates (Figure 6A, upper panel). In sharp contrast, aggregate formation of Tmsb4x KO platelets was significantly decreased at all tested shear rates $\left(150 \mathrm{~s}^{-1}\right.$,

A

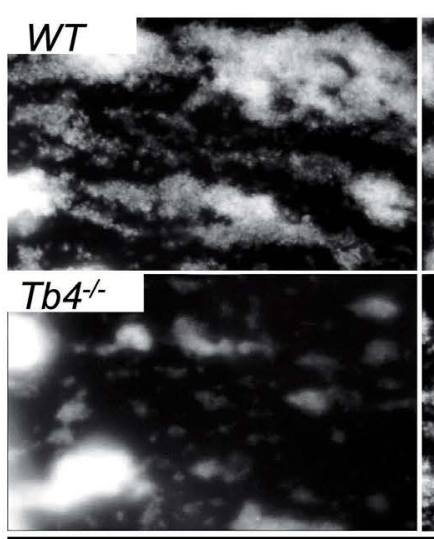

150

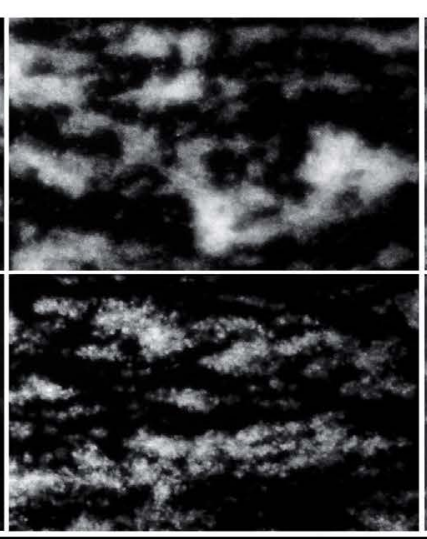

1000 Shear rate $\left[\mathrm{s}^{-1}\right]$

plt. count adjusted $\triangle$

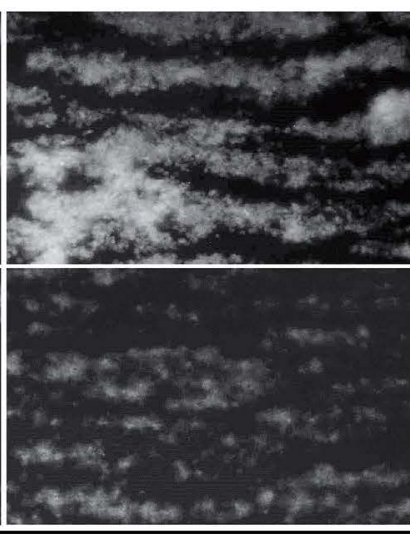

1700

C

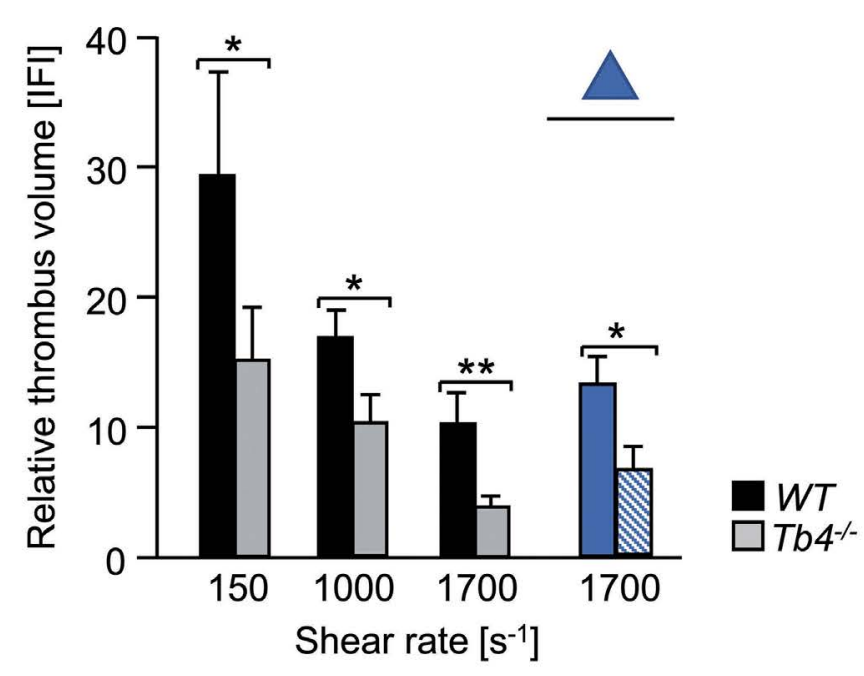

E

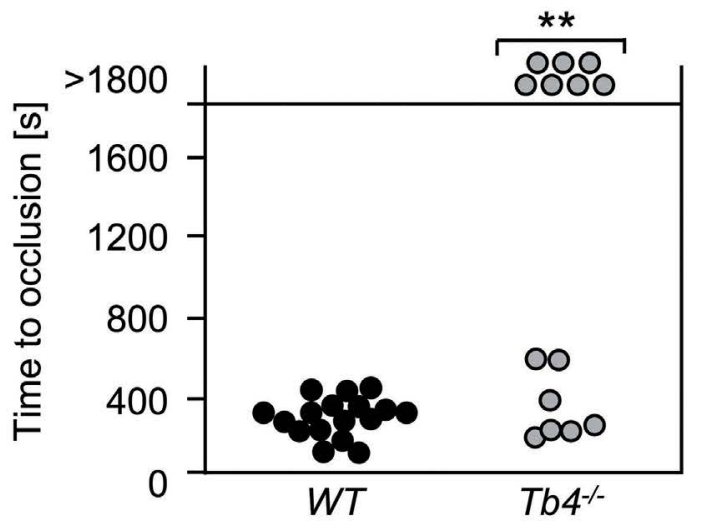

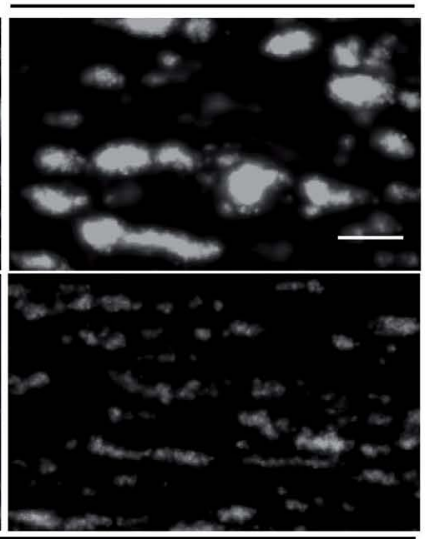

1700
$\mathbf{F}$

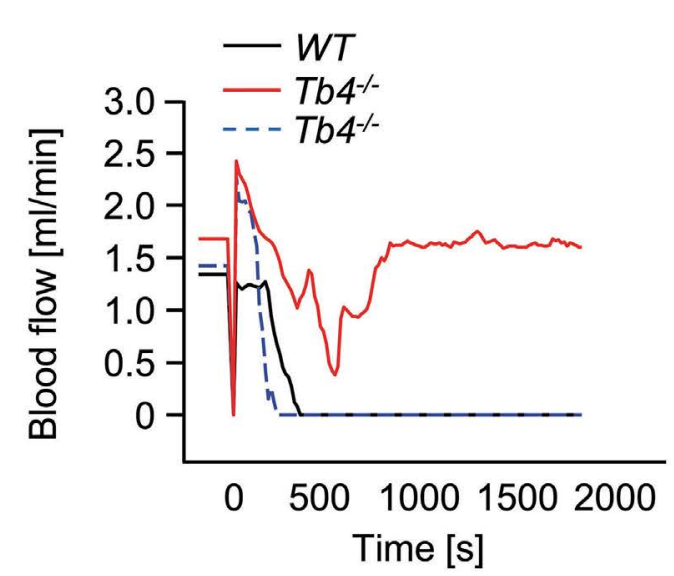

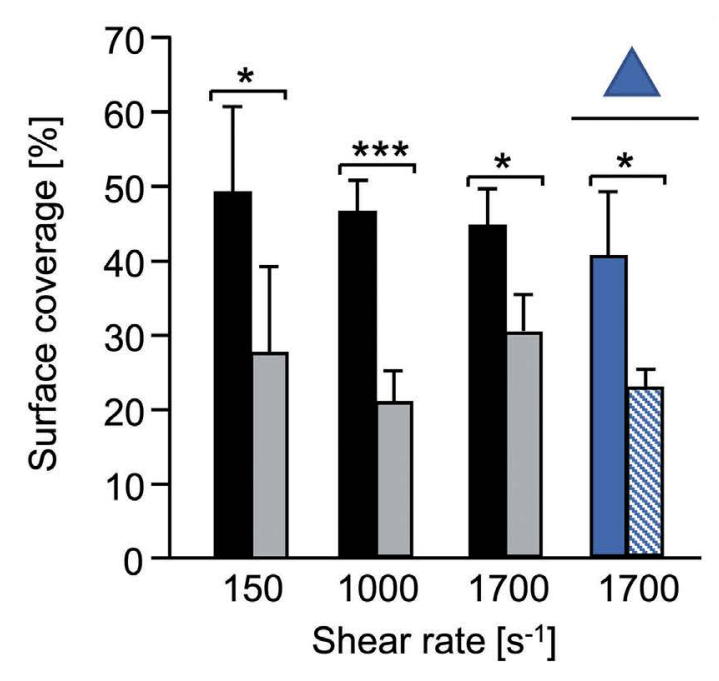

D

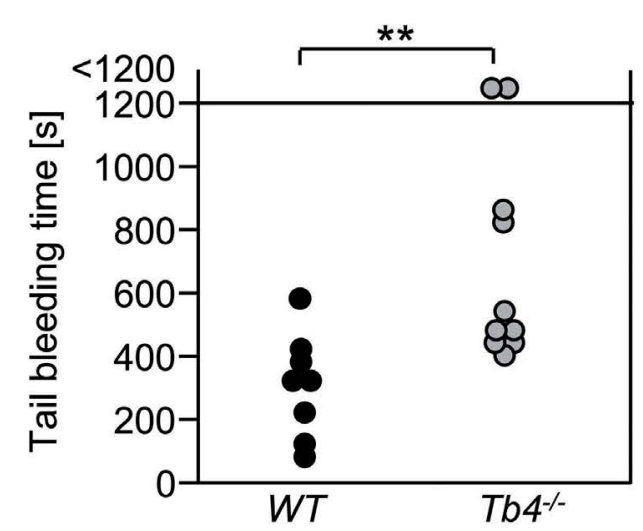

Figure 6. Thymosin $\beta 4$ is required for thrombus formation and stability in vitro and in vivo. (A-C) Assessment of platelet adhesion ( $A, B)$ and aggregate formation (A, C)

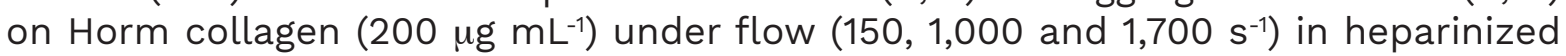
whole blood or platelet-count adjusted blood of WT and Tmsb4x knockout (KO) mice. Values are mean \pm standard deviation (SD) $(n=12)$. Scale bar, $50 \mu \mathrm{m}$. (D) Representative graph of blood flow of one WT and two Tmsb4x KO mice after mechanical injury of the abdominal aorta. (E) Occlusion times after mechanical injury of the abdominal aorta. Data are mean \pm SD of at least 8 mice per group. Fisher's exact test. $* \star P<0.005$. (F) Tail bleeding times in WT and Tmsb4x KO mice (filter paper method). Each symbol represents one individual. Unpaired, two-tailed Student's $t$-test. $* \star P<0.005$. plt.: platelet. 
$\left.\star P<0.05 ; 1,000 \mathrm{~s}^{-1}, * \star \star x<0.001 ; 1,700 \mathrm{~s}^{-1},{ }^{\star} P<0.05\right)$ Figure $6 \mathrm{~A}$ to $\mathrm{C}$ ). In order to exclude that the decreased aggregate formation of Tmsb4x KO platelets was a result of the lower platelet count in these mice, we adjusted platelet counts to WT level, and, strikingly, thrombus volume and platelet surface coverage were still significantly reduced $\left(1,700 \mathrm{~s}^{-1}\right.$, * $P<0.05$; Figure $6 \mathrm{~A}$ to $\mathrm{C}$; platelet count adjusted). Platelet adhesion and aggregate formation under medium and high shear rates are dependent on the interaction between the mechanoreceptor GPIb and immobilized von Willebrand factor (VWF). ${ }^{33}$ In order to investigate a possible involvement of thymosin $\beta 4$ in GPIb-mediated tethering/adhesion, we perfused blood from WT and Tmsb4x KO animals over a vWF-coated surface at high shear $\left(1,700 \mathrm{~s}^{-1}\right)$. However, the number of adherent Tmsb4x KO platelets on immobilized VWF was comparable to the number of WT platelets (Online Supplementary Figure S9), suggesting unaltered GPIb-vWF interaction in mutant platelets.

These results demonstrated that thymosin $\beta 4$ is essential for the formation of stable three-dimensional platelet aggregates on collagen under flow conditions, which appears to be mainly based on a GPVI signaling defect.

\section{Impaired thrombosis and hemostasis in thymosin 34 -deficient mice}

In order to test whether the observed thrombus formation defect translates into an in vivo phenotype, mice were first subjected to a model of occlusive arterial thrombus formation in the mechanically injured aorta, which has been shown to be partly collagen-driven. ${ }^{34}$ After a transient increase directly after injury, blood flow progressively decreased for several minutes in all animals. In all WT mice, this decrease resulted in complete and irreversible occlusion of the artery within 7 min after injury (Figure 6D and E). Out of the 14 tested Tmsb4x KO mice, seven displayed a transient decrease in blood flow, which increased again to normal and led to essentially normal flow rates in the injured vessel at the end of the observation period (30 min; occlusion times; $* \star P<0.01$ ). The other group of KO mice showed a progressive decrease in blood flow, which resulted in full occlusion of the vessel within 8 minutes after injury, almost comparable to that of WT mice. The protection in the subgroup of the mutant mice was most likely due to embolization, a phenomenon that was also observed in a second thrombosis model. Upon $\mathrm{FeCl}_{3}$-induced injury of the mesenteric arterioles, Tmsb4x $\mathrm{KO}$ mice displayed variable time to occlusion that was overall prolonged and displayed a higher rate of embolization (WT embolization: 3/17 arterioles, KO embolization: 12/19 arterioles analyzed) (Online Supplementary Figure S10; Online Supplementary Videos S3 and S4). These results demonstrate that thymosin $\beta 4$ is essential for stable occlusive arterial thrombus formation in vivo.
In order to assess the hemostatic function of Tmsb4x KO platelets, we performed a tail bleeding assay. Notably, tail bleeding times were overall significantly increased in KO mice demonstrating that thymosin $\beta 4$ is also required for normal hemostasis (11.6 $\pm 6.1 \mathrm{~min}$ in $\mathrm{KO}$ mice $v s$. 5.4 2.7 min in $W T ; * * P<0.01$ ) (Figure $6 \mathrm{~F}$ ).

\section{Discussion}

It is already known from studies by others and us that actin-binding proteins have crucial functions during platelet biogenesis and activation. ${ }^{25,26,35}$ The involvement of thymosin $\beta 4$ in $\mathrm{MK} /$ platelet cytoskeletal dynamics, however, has not been assessed to date. Our results reveal that thymosin $\beta 4$ is required for actin remodeling during PPF by MK, and in platelets in response to agonist-induced activation. In line with previous studies on cytoskeletal regulators, the deletion of thymosin $\beta 4$ resulted in a thrombocytopenia with a modest but significant increase in platelet volume, which could be attributed to a defect in PPF. However, in clear contrast to Pfn1 deficiency, ${ }^{24}$ we did not observe premature ectopic release of platelet-like particles in the BM of Tmsb4x $\mathrm{KO}$ mice and the lifespan of peripheral platelets was unaltered in these animals. Instead, analysis of Tmsb4x KO MK revealed abnormal proplatelet shafts and tips, suggesting that defective actin dynamics caused the reduced PPF and thus the observed macrothrombocytopenia. Moreover, we found reduced NMIla and MLC2 levels in Tmsb4x KO platelets, indicating that during PPF NMIla recruitment/enrichment is impaired in the absence of thymosin $\beta 4$. This might contribute to the abnormal appearance of proplatelets as actomyosin contractility plays a crucial role for the process of fragmentation of membrane extensions from MK into the blood flow. ${ }^{30}$

Agonist-induced F-actin assembly was significantly reduced in Tmsb4x KO platelets, which was also reported in Pfn1 deficient platelets, although to a lesser extent. This finding was particularly striking as total F-actin levels were increased in resting Tmsb4x $\mathrm{KO}$ platelets. Sedimentation of the actin cytoskeleton of Tmsb4x KO platelets revealed a marked reduction in the content of G-actin, which is in line with studies suggesting that thymosin $\beta 4$ complexes about half of the monomeric actin in resting cells. ${ }^{36}$ Thus, the robust reduction of the Gactin pool likely accounts for the reduced agonist-induced F-actin assembly in Tmsb4x KO platelets.

Tmsb4x KO platelets also showed accelerated platelet spreading on fibrinogen, which may in part be explained by increased $\mathrm{F}$-actin levels under resting conditions as this process is highly dependent on actin dynamics ${ }^{37}$ and the overload of existing actin filaments might facilitate the formation of filo- and lamellipodia. 
Moreover, clot retraction was significantly enhanced in Tmsb4x KO mice. During blood clot development, platelets interact with fibrin polymers, with contractile force generated internally within the platelet transmitted to the external fibrin clot, resulting in retraction and fibrin clot shrinkage. ${ }^{38}$ Platelet-mediated contractile forces and hence the level of platelet-mediated clot shrinkage are opposed by the rigidity of the 3D fibrin network. ${ }^{38}$ In line with this, Tmsb4x KO platelets showed fewer, less branched and shorter fibrin fibers, indicating that these platelets are able to bend and shorten fibrin fibers faster than WT platelets, which might explain the observed faster clot retraction.

In line with other studies on actin-binding proteins, ${ }^{25,35}$ Tmsb4x KO platelets showed significantly impaired allbb3 integrin activation and degranulation. We speculate that the increased F-actin content in Tmsb4x KO platelets facilitates shape change but disturbs actin dynamics necessary for integrin inside-out signaling during platelet activation and for clot stability. Moreover, these findings point to a specific role of the G-actin/F-actin ratio and the related actin dynamics in $\alpha \mathrm{llb} \beta 3$ activation, granule secretion and aggregation. We assume that the increased ATP release results from an enhanced granule mobilization, which might be attributed to the higher F-actin content of Tmsb4x KO platelets. In case of GPVI agonists the defective GPVI signaling 'overrules' the accelerated degranulation observed with other agonists. This selective defect in ATP release is responsible for the reduction of integrin activation downstream of GPVI, which may be a consequence of reduced Syk phosphorylation. In line with this, Tmsb4x KO platelets showed significantly reduced FcR $\gamma$ chain phosphorylation. Therefore, an explanation for the reduced GPV signaling in Tmsb4x KO platelets might be that lack of thymosin b4 prevents GPVI receptor clustering, ${ }^{39}$ as this presents a mechanism for sustained GPVI signaling essential for prolonged platelet activation trough inhibition of GPVI shedding. ${ }^{40-42}$

Under flow, defective GPVI signaling leads to impaired aggregate formation on collagen and protection from arterial thrombus formation ${ }^{43,44}$ which was also evident in Tmsb4x KO mice. Grb2-deficient platelets show a GPVI-signaling defect that is slightly more severe than that of Tmsb4x KO platelets and consequently form even smaller platelet aggregates under flow conditions. Interestingly, however, arterial thrombus formation following mechanical injury of the abdominal aorta was only mildly affected by the lack of Grb2. ${ }^{45}$ Consequently, the defective GPVI-signaling clearly contributes to the reduced thrombus formation of Tmsb4x KO mice, but other factors are most likely involved. Of note, both FlnA-deficient mouse ${ }^{46}$ as well as human ${ }^{47}$ platelets show comparable defects in integrin signaling, thrombus formation under flow and GPVI signaling. Here, an interaction between Syk and FlnA was shown to regu- late ITAM receptor signaling and platelet function, which could explain the observed defects in FlnA-deficient platelets. However, upon thymosin $\beta 4$ deficiency, the defects seem more complex, as the reduction in integrin activation is not limited to GPVI signaling and Tmsb4x KO platelets show enhanced integrin outside-in signaling. GPVI signaling is critical for the procoagulant activity of platelets ${ }^{48}$ and procoagulant platelets are predominantly localized at the thrombus surface, as a result of their contraction-driven extrusion from the inner core of the thrombus and that such distribution results in surface-enhanced fibrin generation. ${ }^{49}$ Thus, the reduced platelet activation in response to GPVI stimulation in Tmsb4x KO platelets might also affect the fibrin meshwork further destabilizing formed thrombi and thereby enhancing embolization. In addition, to the defective GPVI signaling and enhanced embolization, the accelerated clot retraction is another factor that contribute to the reduced rate of occlusive arterial thrombi observed in Tmsb4x KO mice as compared to WT mice.

Increased tail bleeding times have also been observed in other KO models of actin binding proteins such as $P f n 1^{25}$ and $\operatorname{Cot} 11^{50} \mathrm{KO}$ or $T w f 1 / \operatorname{Cof1}^{26}$ DKO mice. It seems that thrombus stability is reduced in these mouse lines, potentially due to the disturbed actin dynamics. Therefore, we think that the combined defect in the actin-regulating function of thymosin $\beta 4$, the defective GPVI signaling and the reduced thrombus stability led to the increased tail bleeding times.

In summary, we show that thymosin $\beta 4$ controls the polymerization-ready G-actin pool in the megakaryocytic lineage, which inevitably impacts on MK and platelet function. MK displayed abnormal proplatelet shafts and tips, and reduced PPF whereas platelets exhibited accelerated spreading and clot retraction, but reduced GPVI-mediated platelet activation. Our findings highlight that the regulation of the G-actin/F-actin ratio by thymosin $\beta 4$ is not only relevant during platelet biogenesis and activation but is also necessary for the formation of stable thrombi (Online Supplementary Figure S11). Our findings may thus contribute to a better understanding of the molecular pathways orchestrating actin dynamics in cells.

\section{Disclosures}

No conflicts of interest to disclose.

\section{Contributions}

IS, ZN, SB, VG, CG, RAIN, KA and MB acquired data; $B N$ designed the research; IS, SB, ZN, DS, $M B$ and $B N$ analyzed data and wrote the manuscript.

\section{Acknowledgments}

We thank Stefanie Hartmann, Juliana Goldmann and Birgit Midloch for excellent technical assistance and the microscopy platform of the Bioimaging Center (Rudolf Vir- 
chow Center) for providing technical infrastructure and support.

\section{Funding}

This work was supported by the Deutsche Forschungsgemeinschaft (DFG, German Research Foundation; project number 374031971 - TRR 240 and NI 556/11-2 to BN) and the European Union (EFRE - Europäischer Fond für regionale
Entwicklung, Bavaria). MB is supported by an Emmy Noether grant of the DFG (BE5084/3-2). ZN was supported by a grant of the German Excellence Initiative to the Graduate School of Life Sciences, University of Würzburg.

\section{Data-sharing statement}

Please direct requests for original data to the corresponding author: bernhard.nieswandt@virchow.uni-wuerzburg.de.

\section{References}

1. Italiano JE Jr., Lecine P, Shivdasani RA, Hartwig JH. Blood platelets are assembled principally at the ends of proplatelet processes produced by differentiated megakaryocytes. J Cell Biol. 1999; 147(6):1299-1312.

2. Machlus KR, Thon JN, Italiano JE Jr. Interpreting the developmental dance of the megakaryocyte: a review of the cellular and molecular processes mediating platelet formation. Br J Haematol. 2014165(2):227-236.

3. Italiano JE Jr., Patel-Hett S, Hartwig JH. Mechanics of proplatelet elaboration. J Thromb Haemost. 2007;5(Suppl 1):S18-23.

4. Bender M, Thon JN, Ehrlicher AJ, et al. Microtubule sliding drives proplatelet elongation and is dependent on cytoplasmic dynein. Blood. 2015;125(5):860-868.

5. Hartwig JH. Mechanisms of actin rearrangements mediating platelet activation. J Cell Biol. 1992;118(6):1421-1442.

6. Stritt $S$, Nurden P, Turro E, et al. A gain-of-function variant in DIAPH1 causes dominant macrothrombocytopenia and hearing loss. Blood. 2016;127(23):2903-2914.

7. Nurden P, Debili N, Coupry I, et al. Thrombocytopenia resulting from mutations in filamin A can be expressed as an isolated syndrome. Blood. 2011;118(22):5928-5937.

8. Kunishima S, Okuno Y, Yoshida K, et al. ACTN1 mutations cause congenital macrothrombocytopenia. Am J Hum Genet. 2013;92(3):431-438.

9. Goldstein AL, Hannappel E, Kleinman HK. Thymosin beta4: actin-sequestering protein moonlights to repair injured tissues. Trends Mol Med. 2005;11(9):421-429.

10. Low TL, Hu SK, Goldstein AL. Complete amino acid sequence of bovine thymosin beta 4: a thymic hormone that induces terminal deoxynucleotidyl transferase activity in thymocyte populations. Proc Natl Acad Sci U S A. 1981;78(2):1162-1166.

11. Freire M, Hannappel E, Rey M, Freire JM, Kido H, Horecker BL. Purification of thymus mRNA coding for a 16,000-dalton polypeptide containing the thymosin alpha 1 sequence. Proc Natl Acad Sci U S A. 1981;78(1):192-195.

12. Bock-Marquette I, Saxena A, White MD, DiMaio JM, Srivastava D. Thymosin $\beta 4$ activates integrin-linked kinase and promotes cardiac cell migration, survival and cardiac repair. Nature. 2004;432(7016):466-472.

13. Shrivastava S, Srivastava D, Olson EN, DiMaio JM, BockMarquette I. Thymosin beta4 and cardiac repair. Ann N Y Acad Sci. 2010;1194:87-96.

14. Srivastava D, Saxena A, Dimaio JM, Bock-Marquette I. Thymosin beta4 is cardioprotective after myocardial infarction. Ann N Y Acad Sci. 2007;1112:161-70.

15. Huff T, Ballweber E, Humeny A, et al. Thymosin 4 serves as a glutaminyl substrate of transglutaminase. Labeling with fluorescent dansylcadaverine does not abolish interaction with G actin 1. FEBS Lett. 1999;464(1-2):14-20.

16. Zeiler M, Moser M, Mann M. Copy number analysis of the murine platelet proteome spanning the complete abundance range. Mol Cell Proteomics. 2014;13(12):3435-3445.

17. Burkhart JM, Vaudel M, Gambaryan S, et al. The first comprehensive and quantitative analysis of human platelet protein composition allows the comparative analysis of structural and functional pathways. Blood. 2012;120(15):e73-e82.

18. Skruber K, Read TA, Vitriol EA. Reconsidering an active role for G-actin in cytoskeletal regulation. J Cell Sci. 2018;131(1):jcs203760.

19. Xue B, Leyrat C, Grimes JM, Robinson RC. Structural basis of thymosin- $\beta 4 /$ profilin exchange leading to actin filament polymerization. Proc Natl Acad Sci U S A. 2014; 111(43):E4596E4605.

20. Carlier MF, Jean C, Rieger KJ, Lenfant M, Pantaloni D. Modulation of the interaction between $\mathrm{G}$-actin and thymosin beta 4 by the ATP/ADP ratio: possible implication in the regulation of actin dynamics. Proc Natl Acad Sci U S A. 1993;90(11):5034-5038.

21. Hertzog M, van Heijenoort C, Didry D, et al. The betathymosin/WH2 domain; structural basis for the switch from inhibition to promotion of actin assembly. Cell. 2004;117(5):611-623.

22. Didry D, Cantrelle FX, Husson C, et al. How a single residue in individual beta-thymosin/WH2 domains controls their functions in actin assembly. EMBO J. 2012;31(4):1000-1013.

23. Goldschmidt-Clermont PJ, Furman MI, Wachsstock D, Safer D, Nachmias VT, Pollard TD. The control of actin nucleotide exchange by thymosin beta 4 and profilin. A potential regulatory mechanism for actin polymerization in cells. Mol Biol Cell. 1992;3(9):1015-1024.

24. Bender M, Stritt S, Nurden P, et al. Megakaryocyte-specific Profilin1-deficiency alters microtubule stability and causes a Wiskott-Aldrich syndrome-like platelet defect. Nat Commun. 2014;5:4746.

25. Stritt S, Birkholz I, Beck S, et al. Profilin 1-mediated cytoskeletal rearrangements regulate integrin function in mouse platelets. Blood Advs. 2018;2(9):1040-1045.

26. Becker IC, Scheller I, Wackerbarth LM, et al. Actin/microtubule crosstalk during platelet biogenesis in mice is critically regulated by Twinfilin1 and Cofilin1. Blood Adv. 2020;4(10):2124-2134.

27. Heib T, Gross C, Müller ML, Stegner D, Pleines I. Isolation of murine bone marrow by centrifugation or flushing for the analysis of hematopoietic cells - a comparative study. Platelets. 2021;32(5):601-607.

28. Spindler M, van Eeuwijk JMM, Schurr Y, et al. ADAP deficiency impairs megakaryocyte polarization with ectopic proplatelet release and causes microthrombocytopenia. Blood. 2018;132(6):635-646.

29. Sabri S, Foudi A, Boukour S, et al. Deficiency in the Wiskott- 
Aldrich protein induces premature proplatelet formation and platelet production in the bone marrow compartment. Blood. 2006;108(1):134-140.

30. Bächer C, Bender M, Gekle S. Flow-accelerated platelet biogenesis is due to an elasto-hydrodynamic instability. Proc Natl Acad Sci U S A. 2020;117(32):18969-18976.

31. Cramer LP, Briggs LJ, Dawe HR. Use of fluorescently labelled deoxyribonuclease I to spatially measure G-actin levels in migrating and non-migrating cells. Cell Motil Cytoskeleton. 2002;51(1):27-38.

32. Campbell RA, Overmyer KA, Selzman $\mathrm{CH}$, Sheridan BC, Wolberg AS. Contributions of extravascular and intravascular cells to fibrin network formation, structure, and stability. Blood. 2009;114(23):4886-4896.

33. Turitto VT, Weiss HJ, Baumgartner HR. The effect of shear rate on platelet interaction with subendothelium exposed to citrated human blood. Microvasc Res. 1980;19(3):352-365.

34. Bender M, Hagedorn I, Nieswandt B. Genetic and antibodyinduced glycoprotein VI deficiency equally protects mice from mechanically and FeCl3-induced thrombosis. J Thromb Haemost. 2011;9(7):1423-1426.

35. Stritt S, Beck S, Becker IC, et al. Twinfilin 2a regulates platelet reactivity and turnover in mice. Blood. 2017;130(15):1746-1756.

36. Mannherz HG, Hannappel E. The beta-thymosins: intracellular and extracellular activities of a versatile actin binding protein family. Cell Motil Cytoskeleton. 2009;66(10):839-851.

37. Varga-Szabo D, Braun A, Nieswandt B. Calcium signaling in platelets. J Thromb Haemost. 2009;7(7):1057-1066.

38. Samson AL, Alwis I, Maclean JAA, et al. Endogenous fibrinolysis facilitates clot retraction in vivo. Blood. 2017; 130(23):2453-2462.

39. Poulter NS, Pollitt AY, Owen DM, et al. Clustering of glycoprotein VI (GPVI) dimers upon adhesion to collagen as a mechanism to regulate GPVI signaling in platelets. J Thromb Haemost. 2017;15(3):549-564.
40. Pallini C, Pike JA, O'Shea C, et al. Immobilized collagen prevents shedding and induces sustained GPVI clustering and signaling in platelets. Platelets. 2021;32(1):59-73.

41. Moroi M, Jung SM. Platelet glycoprotein VI: its structure and function. Thromb Res. 2004;114(4):221-233.

42. Berlanga O, Bori-Sanz T, James JR, et al. Glycoprotein VI oligomerization in cell lines and platelets. J Thromb Haemost. 2007;5(5):1026-1033.

43. Massberg S, Gawaz M, Grüner S, et al. A crucial role of glycoprotein $\mathrm{VI}$ for platelet recruitment to the injured arterial wall in vivo. J Exp Med. 2003;197(1):41-49.

44. Nieswandt B, Brakebusch C, Bergmeier W, et al. Glycoprotein VI but not alpha2beta1 integrin is essential for platelet interaction with collagen. EMBO J. 2001;20(9):2120-2130.

45. Dutting S, Vogtle T, Morowski M, et al. Growth factor receptorbound protein 2 contributes to (hem)immunoreceptor tyrosine-based activation motif-mediated signaling in platelets. Circ Res. 2014;114(3):444-453.

46. Falet $H$, Pollitt AY, Begonja AJ, et al. A novel interaction between FlnA and Syk regulates platelet ITAM-mediated receptor signaling and function. J Exp Med. 2010;207(9):1967-1979.

47. Berrou E, Adam F, Lebret M, et al. Heterogeneity of platelet functional alterations in patients with filamin A mutations. Arterioscler Thromb Vasc Biol. 2013;33(1):e11-18.

48. Swieringa F, Spronk HMH, Heemskerk JWM, van der Meijden PEJ. Integrating platelet and coagulation activation in fibrin clot formation. Res Pract Thromb Haemost. 2018;2(3):450-460.

49. Nechipurenko DY, Receveur N, Yakimenko AO, et al. Clot contraction drives the translocation of procoagulant platelets to thrombus surface. Arterioscler Thromb Vasc Biol. 2019;39(1):37-47.

50. Scheller I, Stritt S, Beck S, et al. Coactosin-like 1 integrates signaling critical for shear-dependent thrombus formation in mouse platelets. Haematologica. 2020;105(6):1667-1676. 